Z Rheumatol 2018 77 (Suppl 2):S35-S53 https://doi.org/10.1007/s00393-018-0481-y Online publiziert: 3. Juli 2018

(c) Deutsche Gesellschaft für Rheumatologie e.V. Published by Springer Medizin Verlag $\mathrm{GmbH}$. All rights reserved 2018

CrossMark

C. Fiehn ${ }^{1,5} \cdot$ J. Holle ${ }^{1,6} \cdot$ C. Iking-Konert ${ }^{1,7} \cdot$ J. Leipe $^{1,8} \cdot$ C. Weseloh ${ }^{1} \cdot$ M. Frerix ${ }^{1,9}$. R. Alten ${ }^{1,10} \cdot$ F. Behrens ${ }^{1,11,12} \cdot$ C. Baerwald ${ }^{1,13} \cdot$ J. Braun $^{1,14} \cdot$ H. Burkhardt ${ }^{1,11,12}$. G. Burmester, ${ }^{1,15} \cdot$ J. Detert ${ }^{1,15} \cdot$ M. Gaubitz ${ }^{1,16} \cdot$ A. Gause $^{1,17} \cdot$ E. Gromnica-Ihle . $^{3}$ H. Kellner ${ }^{1,18,19} \cdot$ A. Krause ${ }^{1,20} \cdot$ J. Kuipers ${ }^{1,21} \cdot$ H.-M. Lorenz ${ }^{1,22,23} \cdot$ U. Müller-Ladner ${ }^{1,9} \cdot$ M. Nothacker ${ }^{4} \cdot$ H. Nüsslein ${ }^{1,24} \cdot$ A. Rubbert-Roth ${ }^{1,25} \cdot$ M. Schneider ${ }^{1,26} \cdot$ H. Schulze-

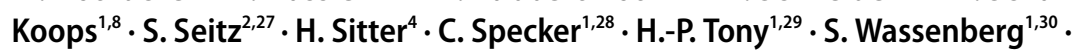
J. Wollenhaupt ${ }^{1,31} \cdot$ K. Krüger ${ }^{1,32}$

${ }^{1}$ Deutsche Gesellschaft für Rheumatologie (DGRh), Berlin, Deutschland; ${ }^{2}$ Deutsche Gesellschaft für Orthopädie und Orthopädische Chirurgie e. V. (DGOOC) und Deutsche Gesellschaft für Orthopädische Rheumatologie (DGORh), Hamburg, Deutschland; ${ }^{3}$ Deutsche Rheuma-Liga Bundesverband e.V., Bonn, Deutschland; ${ }^{4}$ Arbeitsgemeinschaft der Wissenschaftlichen Medizinischen Fachgesellschaften e.V. (AWMF), Berlin, Deutschland; ${ }^{5}$ Praxis für Rheumatologie, Tätigkeitsschwerpunkt Klinische Immunologie und Belegarzteinheit der ViDia-Kliniken Karlsruhe am Medical Center Baden-Baden, Baden-Baden, Deutschland; ${ }^{6}$ Rheumazentrum Schleswig-Holstein Mitte, Neumünster, Deutschland; ${ }^{7}$ Klinik für Nephrologie und Rheumatologie, Med. Klinik III, Universitätsklinik, Hamburg Eppendorf (UKE), Hamburg, Deutschland; ${ }^{8}$ Sektion Rheumatologie und Klinische Immunologie, Medizinische Klinik und Poliklinik IV Ludwig-Maximilians-Universität, München, Deutschland; ${ }^{9}$ Abteilung für Rheumatologie und Klinische Immunologie, Campus Kerckhoff, Justus-Liebig-Universität Gießen, Bad Nauheim, Deutschland; ${ }^{10}$ Abteilung Innere Medizin II, Rheumatologie, Klinische Immunologie, Osteologie, Schlosspark-Klinik, Charité - Universitätsmedizin Berlin, Berlin, Deutschland; "Abteilung Rheumatologie, Universitätsklinikum Frankfurt am Main, Goethe Universität Frankfurt am Main, Frankfurt am Main, Deutschland; ${ }^{12}$ Fraunhofer Institut IME, Translationale Medizin und Pharmakologie, Frankfurt am Main, Deutschland; ${ }^{13}$ Sektion Rheumatologie, Department für Innere Medizin, Neurologie und Dermatologie, Universitätsklinikum Leipzig, Leipzig, Deutschland; ${ }^{14}$ Rheumazentrum Ruhrgebiet, Herne, Deutschland; ${ }^{15}$ Medizinische Klinik mit Schwerpunkt Rheumatologie und Klinische Immunologie, Charité Universitätsmedizin Berlin, Campus Mitte (CCM), Berlin, Deutschland; ${ }^{16}$ Akademie für Manuelle Therapie an der WWU Münster, Münster, Deutschland; ${ }^{17}$ Rheumapraxis Bad Bramstedt, Bad Bramstedt, Deutschland; ${ }^{18}$ Schwerpunktpraxis für Rheumatologie und Gastroenterologie, München, Deutschland; ${ }^{19} \mathrm{Abteilung}$ Rheumatologie, Krankenhaus Neuwittelsbach, München, Deutschland; ${ }^{20} \mathrm{Klinik}$ für Rheumatologie und Klinische Immunologie, Immanuel Krankenhaus Berlin, Berlin, Deutschland; ${ }^{21}$ Klinik für internistische Rheumatologie, Rotes Kreuz Krankenhaus Bremen gGmbH, Bremen, Deutschland; ${ }^{22}$ Medizinische Universitätsklinik V: Hämatologie, Onkologie, Rheumatologie, Universitätsklinikum Heidelberg, Heidelberg, Deutschland; ${ }^{23}$ ACURA Rheumazentrum Baden-Baden, Baden-Baden, Deutschland; ${ }^{24}$ Rheumatologische Schwerpunktpraxis, Nürnberg, Deutschland; ${ }^{25}$ Klinik für Rheumatologie, Kantonsspital St. Gallen, St. Gallen, Schweiz; ${ }^{26}$ Poliklinik, Funktionsbereich und Hiller Forschungszentrum für Rheumatologie, Heinrich-Heine Universität Düsseldorf, Universitätsklinikum Düsseldorf, Düsseldorf, Deutschland; ${ }^{27}$ Klinik für Orthopädie, Klinikum Hochsauerland, Marienhospital, Arnsberg, Deutschland; ${ }^{28}$ Klinik für Rheumatologie und klinische Immunologie, St. Josef Krankenhaus Essen-Werden, Universitätsmedizin Essen, Essen, Deutschland; ${ }^{29}$ Abteilung für Rheumatologie und klinische Immunologie, Zentrum für Innere Medizin, Universitätsklinikum Würzburg, Würzburg, Deutschland; ${ }^{30}$ Rheumazentrum Ratingen, Ratingen, Deutschland; ${ }^{31}$ Rheumatologikum Hamburg, Hamburg, Deutschland; ${ }^{32}$ Rheumatologisches Praxiszentrum, München, Deutschland

\title{
S2e-Leitlinie: Therapie der rheumatoiden Arthritis mit krankheitsmodifizierenden Medikamenten
}




\section{Zusatzmaterial online}

Die Online-Version dieses Beitrags (https://doi.org/10.1007/s00393-0180481-y) enthält weitere, detaillierte Informationen zur systematischen Literaturrecherche sowie Evidenztabellen und tabellarische Übersichten der risk-of-bias-Bewertung der Evidenz. Beitrag und Zusatzmaterial stehen Ihnen im elektronischen Volltextarchiv auf http:// www.springermedizin.de/zeitschrift-fuerrheumatologie zur Verfügung. Sie finden das Zusatzmaterial am Beitragsende unter „Supplementary Material“.

\section{Infobox \\ AWMF-Leitlinien Register Nummer: 060-004 Entwicklungsstufe: S2e \\ Convenor/Leitliniensekretariat: \\ Prof. Dr. Christoph Fiehn \\ Inhaltlicher Stand: April 2018, \\ Gültigkeit der Leitlinie: bis April \\ 2023 \\ Verabschiedung der Leitlinie durch den Vorstand der DGRh März 2018 \\ Besonderer Hinweis: Die Medizin unterliegt einem fortwährenden Entwicklungsprozess, sodass alle Angaben, insbesondere zu therapeutischen Verfahren, immer nur dem Wissensstand zur Zeit der Drucklegung der Leitlinie entsprechen können. Hinsichtlich der angegebenen Empfehlungen zur Therapie wurde die größtmögliche Sorgfalt beachtet. Der Benutzer selbst bleibt verantwortlich für jede diagnostische und therapeutische Applikation, Medikation und Dosierung.}

\section{Einleitung}

Moderne Therapiestrategien mit krankheitsmodifizierenden Medikamenten haben das Bild der rheumatoiden Arthritis (RA) in neuerer Zeit stark verändert. Der Fortschritt ist u. a. ablesbar in geringerer radiologischer Progression, aber auch verbesserter Funktionalität von $\mathrm{Pa}$ tienten mit RA [31, 33, 128]. Zudem reduzieren moderne Therapien signifikant Arbeitsunfähigkeit, Erwerbsunfähigkeit [109], kardiovaskuläre Morbidität [8] und Gesamtmortalität [69] bei RAPatienten und sind dabei kosteneffektiv [126].

Voraussetzung dieser positiven therapeutischen Entwicklung war das bessere pathophysiologische Verständnis der RA als entzündliche Systemerkrankung mit im Vordergrund stehender proliferativer
Synovitis und inhärentem gelenkzerstörerischem Potential. Als logische Konsequenz folgte die Notwendigkeit der frühen und möglichst vollständigen und nachhaltigen Unterdrückung der Entzündung bei der RA. Neue Klassifikationskriterien der RA [5] erlauben die Erkrankung früher zu detektieren und das Konzept des Treat-to-Target (T2T) mit dem Ziel der Remission umzusetzen [92]. Diese Remission ist nun, deutlich strenger als früher, als nahezu vollständige Entzündungs- und Beschwerdefreiheit definiert ([30]; siehe Glossar, - Tab. 1). Die Entwicklung neuer hochwirksamer und zielgerichteter Medikamente hat diese Entwicklung weiter begünstigt. Das Ziel der hier vorgestellten Leitlinie ist es, eine evidenzund konsensbasierte Empfehlung für eine bestmögliche Therapie der RA in Deutschland nach dem aktuellen Stand der Wissenschaft zu geben. Sie baut auf der S1-Leitlinie der Deutschen Gesellschaft für Rheumatologie (DGRh) von 2012 auf [65].

\section{Methoden}

Bei der Konzeption und der Durchführung der Leitlinie wurde den Empfehlungen der European League Against Rheumatism (EULAR) [111] und des Manuals der Arbeitsgemeinschaft der wissenschaftlichen medizinischen Fachgesellschaften (AWMF) (www.awmf. org/leitlinien) für die Erstellung von Empfehlungen und Leitlinien gefolgt. Es wurde eine systematische Literaturrecherche durchgeführt (C.W.), die detailliert im Zusatzmaterial online beschrieben ist.

Die Sichtung der Literatur und die Erarbeitung von Vorschlägen für die Konsenstreffen erfolgte in 5 Arbeitsgruppen (AG Therapiealgorithmus, AG Treat-toTarget, AG Deeskalation, AG Personalisierte Medizin und AG JAK-Inhibitoren) mit den jeweiligen Leitern K. K, C. F., J.H., C. I.-K. sowie J.L.

Die Empfehlungen wurden in einem strukturierten Konsensprozess erarbeitet. Nach einem ersten konstituierenden Treffen wurde dieser Prozess in drei weiteren Treffen jeweils von externen, speziell geschulten Moderatoren (M.N. und
H.S.) der AWMF ohne Interessenkonflikte geleitet. Dabei wurden jeweils Empfehlungsgrade nach der Systematik des Oxford Centre for Evidence-Based Medicine von 2009 (www.cebm.net) vergeben.

Vor Beginn des Konsensprozesses wurden die Interessenkonflikte der Teilnehmer mit einem von der AWMF entworfenen Fragebogen erfragt. Die dabei angegebenen Interessenkonflikte wurden durch 3 von der DGRh benannte Ombudspersonen (E. Genth, Aachen, W. Gross, Lübeck, H.-H. Peter, Freiburg) begutachtet. Wenn Interessenkonflikte vorlagen die den Ombudspersonen als bedeutend erschienen, wurde beschlossen, dass sich die jeweiligen Mitglieder der Gruppe bei den Konsens-Abstimmungen der Stimme zu enthalten hatten. Dies war der Fall, z.B. wenn vermutet wurde, dass Teilnehmer durch Beteiligungen an Firmen zur Durchführung von klinischen Studien oder aber durch sonstige direkte wirtschaftliche Interessen nicht die notwendige Unabhängigkeit haben könnten.

Der Risk of Bias (RoB) wurde nach der Methode ACROBAT-NRSI der Cochrane Bias Methods Group (methods. cochrane.org/bias/home und www. riskofbias.info) evaluiert (M.F.). Evidenztabellen wurden in modifizierter Form nach dem Standard der Evidence Tables Working Group des Guidelines International Network erarbeitet (www. g-i-n.net). In den jeweiligen Arbeitsgruppen wurden die dafür wichtigsten Referenzen der entsprechenden Themen ausgewählt. Die RoB-Tabellen lagen bei den Konsenstreffen und Diskussionen der jeweiligen Themen vor. RoB- und Evidenztabellen sind im Zusatzmaterial online einzusehen.

\section{Ergebnisse}

\section{Allgemeine Prinzipien der Therapie}

\section{Übergeordnete Prinzipien}

Die übergeordneten Prinzipien A-F sind in - Tab. 2 aufgelistet.

Alle Prinzipien mit Ausnahme von D wurden einstimmig beschlossen. Bei der Zuständigkeit des internistischen Rheumatologen für das Management der 
Z Rheumatol 2018 77 (Suppl 2):S35-S53 https://doi.org/10.1007/s00393-018-0481-y

(c) Deutsche Gesellschaft für Rheumatologie e.V. Published by Springer Medizin Verlag GmbH. All rights reserved 2018

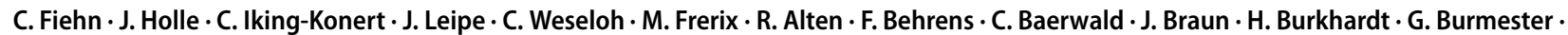

J. Detert · M. Gaubitz · A. Gause · E. Gromnica-Ihle · H. Kellner · A. Krause · J. Kuipers · H.-M. Lorenz · U. Müller-Ladner · M. Nothacker · H. Nüsslein · A. Rubbert-Roth - M. Schneider · H. Schulze-Koops · S. Seitz · H. Sitter · C. Specker · H.-P. Tony · S. Wassenberg · J. Wollenhaupt · K. Krüger

\section{S2e-Leitlinie: Therapie der rheumatoiden Arthritis mit krankheitsmodifizierenden Medikamenten}

\section{Zusammenfassung}

Hintergrund. Medikamentöse Therapiestrategien zur Behandlung der rheumatoiden Arthritis sind entscheidend für den Langzeitverlauf. Sie dienen dem Ziel, durch frühe und konsequente Unterdrückung der Entzündung Gelenkzerstörung zu verhindern und damit die Funktion zu erhalten.

Ziel der Arbeit. Erarbeitung eines Konsenses für evidenzbasierte Empfehlungen zur Behandlung der rheumatoiden Arthritis mit krankheitsmodifizierenden Medikamenten in Deutschland.

Methoden. Nach einer systematischen Literatursuche wurde ein strukturierter Konsensprozess durchgeführt.

Ergebnisse. Sechs übergeordnete Prinzipien und 10 Empfehlungen fassen die Ergebnisse des Konsensprozesses zusammen. Verschiedene Punkte sind gegenüber der Fassung von
2012 neu, so die differenzierte Anpassung des Therapieregimes nach Zeitpunkt und Ausmaß des Ansprechens, das Therapieziel Remission gemessen mithilfe des simplified disease activity index (SDAI) wie auch der potentielle Einsatz zielgerichteter synthetischer DMARDs (tsDMARDs), der JAK-Inhibitoren sowie Empfehlungen zur Deeskalation nach dem Erreichen einer anhaltenden Remission. Wie bisher steht Methotrexat (MTX) im Mittelpunkt der Therapie zu Beginn und als Kombinationspartner im weiteren Verlauf. Die Kombination mehrerer konventioneller synthetischer DMARDs, oder, bei ungünstigen prognostischen Faktoren, der Einsatz von biologischen oder tsDMARDs kommen nach dem Algorithmus bei nicht ausreichendem Ansprechen auf Methotrexat zur Anwendung. Empfehlungen für die Deeskalation der
Therapie mit Glukokortikoiden und konsekutiv gegebenenfalls auch DMARDs geben Hilfe für den Umgang mit Patienten, die eine anhaltende Remission erreicht haben. Zusammenfassung. Die neue S2-Leitlinie gibt Empfehlungen für die Therapie der RA nach dem Prinzip des "Treat-toTarget" (T2T) mit etablierten und neuen krankheitsmodifizierenden Medikamenten (DMARDs), einschließlich der Biologika und JAK-Inhibitoren und macht Vorschläge zur Deeskalation nach Erreichen einer anhaltenden Remission.

Schlüsselwörter

Rheumatoide Arthritis · Leitlinie · Evidenz . Konsens · DMARD

\section{S2e guideline: treatment of rheumatoid arthritis with disease-modifying drugs}

\section{Abstract}

Background. Medication-based strategies to treat rheumatoid arthritis are crucial in terms of outcome. They aim at preventing joint destruction, loss of function and disability by early and consistent inhibition of inflammatory processes.

Objective. Achieving consensus about evidence-based recommendations for the treatment of rheumatoid arthritis with disease-modifying anti-rheumatic drugs in Germany.

Methods. Following a systematic literature research, a structured process among expert rheumatologists was used to reach consensus. Results. The results of the consensus process can be summed up in 6 overarching principles and 10 recommendations. There are several new issues compared to the version of 2012 such as differentiated adjustments to the therapeutic regime according to time point and extent of treatment response, the therapeutic goal of achieving remission as assessed by means of the simplified disease activity index (SDAI) as well as the potential use of targeted synthetic DMARDs (JAK inhibitors) and suggestions for a deescalating in case of achieving a sustained remission. Methotrexate still plays the central role at the beginning of the treatment and as a combination partner in the further treatment course. When treatment response to methotrexate is inadequate, either switching to or combining with another conventional synthetic DMARD is an option in the absence of unfavourable prognostic factors. Otherwise biologic or targeted synthetic DMARDs are recommended according to the algorithm.

Rules for deescalating treatment with glucocorticoids and-where applicable-DMARDs give support for the management of patients who have reached a sustained remission. Discussion. The new guidelines set up recommendations for RA treatment in accordance with the treat-to-target principle. Modern disease-modifying drugs, now including also JAK inhibitors, are available in an algorithm.

\section{Keywords}

Rheumatoid arthritis · Guideline - Evidence . Consensus · DMARD
RA (übergeordnetes Prinzip D) bestand ebenfalls eine hohe Übereinstimmung, es gab jedoch ein Minderheitsvotum (S.S.) in der Konsensgruppe das Wort „internistisch“ zu streichen.

\section{Therapiealgorithmus und Empfehlungen}

Der empfohlene Therapiealgorithmus, wie er in den Konsensgruppen erarbeitet wurde, ist in Abb. 1a-c dargestellt.

\section{Empfehlung 1}

Sobald die Diagnose einer RA gestellt ist, soll eine Therapie mit DMARD begonnen werden. (Empfehlungsgrad A)

Der frühestmögliche Beginn einer DMARD-Therapie bei neu diagnostizierter RA (optimalerweise innerhalb von 12 Wochen nach Symptombeginn) wirkt sich positiv auf die Parameter Remission, therapeutisches Ansprechen, 


\section{Tab. 1 Glossar}

\begin{tabular}{|c|c|}
\hline Begriff & Definition \\
\hline \multicolumn{2}{|l|}{ Status der Krankheitsaktivität } \\
\hline \multirow[t]{2}{*}{ Remission [29] } & $\begin{array}{l}\text { ACR-EULAR Boole'sche Kriterien von 2011: SJC, TJC, CRP (mg/dl) } \\
\text { und Patientenurteil (PG), jeweils } \leq 1\end{array}$ \\
\hline & Composite-Score-basierte Definition: SDAI $(\leq 3,3)$ \\
\hline \multirow{4}{*}{$\begin{array}{l}\text { Niedrige Krankheitsaktivität } \\
\text { (LDA: low disease activity) }\end{array}$} & Gemäß eines validierten Composite Scores: \\
\hline & SDAI >3,3-11 \\
\hline & $\mathrm{CDAI}>2,8-10$ \\
\hline & DAS2 $28 \geq 2,6-<3,2$ \\
\hline \multirow{4}{*}{$\begin{array}{l}\text { Moderate und/oder hohe } \\
\text { Krankheitsaktivität }\end{array}$} & Gemäß eines validierten Composite Scores: \\
\hline & SDAl: moderat $>11-26$; hoch: $>26$ \\
\hline & CDAl: moderat $>10-22 ;$ hoch: $>22$ \\
\hline & DAS28: moderat $3,2-<5,1 ;$ hoch: $\geq 5,1$ \\
\hline
\end{tabular}

Response (Verbesserung)

Flare (Schub)

DMARD Nomenklatur [95]

(Konventionelle)

synthetische DMARDs

Biologische DMARDs

Medikamente

Niedrig-dosiertes Glukokortikoid („low-dose-Steroid")

TNF-Inhibitoren

Sonstige biologische DMARDs/„Biologika“ mit anderem

Wirkmechanismus ${ }^{\mathrm{a}, \mathrm{b}}$

JAK-Inhibitoren (JAKi)

ACPA

Engl.: anti citrullinated peptide/protein antibodies
Mind. $50 \%$ Verbesserung in einem Composite Score (z. B. DAS28)

Verlust von Remission oder niedriger Krankheitsaktivität durch Anstieg der Krankheitsaktivität (gemessen mit Composite Scores) über eine tägliche Variation der Beschwerden hinaus

Conventional synthetic DMARDs (csDMARDs), z. B. MTX, Leflunomid, Sulfasalazin, Hydroxychloroquin

Targeted synthetic DMARDs (tsDMARDs), z. B. Baricitinib oder Tofacitinib

Biological originator DMARDs (boDMARDs)

Biosimilar DMARDs (bsDMARDs) (Kommentar siehe auch • Infobox 1)

Adalimumab (bs in 2018 erwartet), Certolizumab, Etanercept (bo und bs), Golimumab, Infliximab (bo und bs) ${ }^{a}$

IL-6-Rezeptor-Inhibitoren: Sarilumab und Tocilizumab

Anti-CD20: Rituximab (bo und bs)

Anti-CTLA-4: Abatacept

Baricitinib und Tofacitinib ${ }^{\mathrm{a}}$

Am häufigsten i.S. von Anti-CCP-Antikörpern, aber auch seltener Antikörper wie anti-MCV (anti-mutiertes citrulliniertes Vimentin)
Laut EULAR $<7,5 \mathrm{mg}$ Prednison

ACR American College of Rheumatology, BSG Blutkörperchensenkungsgeschwindigkeit, CDAI clinical disease activity score (0-76), Composite Score validierter, zusammengesetzter Krankheitsaktivitätsindex (insbes. DAS28, SDAl, CDAI), CRP C-reaktives Protein, DMARDs disease-modifying antirheumatic drugs, EULAR European League Against Rheumatism, SDAl simplified disease activity score (0-86), DAS disease activity score $28(0-9,4)$, SJC swollen joint count (Anzahl der geschwollenen Gelenke), TJC tender joint count (Anzahl der druckschmerzhaften Gelenke), SDAI simplified disease activity score (0-86), DAS28 disease activity score $28(0-9,4)$

âStand März 2018

bIL-1RA-Therapie (Anakinra) hat trotz Zulassung i. d. R. keinen Stellenwert mehr bei der Behandlung der RA

Funktionsstatus und radiologische Progression aus und wird in einer großen Zahl kontrollierter Studien belegt $(\mathrm{Zu}$ sammenfassung und Übersicht unter [16]). Gegenwärtig ist noch unklar, ob der Beginn einer DMARD-Therapie überhaupt die Diagnose einer gesicherten RA erfordert und ob ein noch früherer Therapiebeginn zusätzlich den Langzeitverlauf positiv beeinflusst. Eine weitere Diskussion dazu ist künftig erforderlich. Die kontrollierte IMPROVED-
Studie behandelte sowohl Patienten mit früher gesicherter RA und (noch) undifferenzierter Arthritis. In dieser Studie wurden durchweg hohe Remissionsraten [52] (LoE 1b) erreicht. Allerdings konnte auf die Frage, wie groß der Vorteil der Behandlung vor Diagnosesicherung tatsächlich ist, noch keine Antwort gefunden werden. Der Einsatz von Methotrexat (MTX) vs. Placebo bei undifferenzierter Arthritis („wahrscheinliche RA“) führte in der PROMPT-Studie nach 30 Monaten zur Verzögerung der radiologischen Progression und des Übergangs in eine gesicherte RA [116] (LoE 1b). Nach fünf Jahren gab es zwischen beiden Gruppen jedoch keine Unterschiede im Outcome [110] (LoE 2). Ein DMARD-Beginn bei vermuteter, aber noch nicht gesicherter RA ist somit noch nicht generell $\mathrm{zu}$ empfehlen, wenn auch im Einzelfall gute Gründe dafür sprechen können. Weitere Studien sind zur Eingrenzung der Subgruppe von Patienten notwendig, die von einer solchen sehr frühen Therapie profitiert (s. Forschungs-Agenda).

\section{Empfehlung 2}

Das Ziel der Therapie ist das Erreichen und die Erhaltung einer Remission. (Empfehlungsgrad A)

Es besteht ein Konsens zur Messung der klinischen Krankheitsaktivität mit einem Composite-Score bei T2T. Dabei können z.B. der DAS28, CDAI oder SDAI genutzt werden (siehe Glossar, - Tab. 1) (Empfehlungsgrad A). Das Ziel von T2T ist, einen Krankheitsaktivitätsscore zu erreichen, der einer Remission entspricht. Der Vorteil dieser Vorgehensweise wurde durch eine Vielzahl von klinischen Studien belegt [103]. Die inzwischen nicht mehr zeitgemäßen alten EULAR-Response-Kriterien [117] haben eine zu hohe klinische Aktivität und das Fortschreiten der Gelenkzerstörung zugelassen. Diese Beobachtungen wurden z.B. in der SWEFOT-Studie erhoben [84]. Daher ist es Konsens, dass die neuen EULAR/ACR-Kriterien für die Remission (Boole'sche Kriterien oder Index-basierte Kriterien i.S. des SDAI, siehe Glossar) [30] das Ziel des T2T sein sollten (Empfehlungsgrad A). Nach Meinung der Konsensgruppe ist in der Praxis des Rheumatologen der SDAI 
A. Die Behandlung der RA sollte die bestmögliche medizinische Betreuung des Patienten zum Ziel haben und auf gemeinsamen Entscheidungen durch den Patienten/-in und den Rheumatologen/-in basieren

B. Die RA ist eine schwere Erkrankung, die mit hohen direkten und indirekten Kosten verbunden ist. Dies sollte durch den behandelnden Rheumatologen bei seinen Entscheidungen berücksichtigt werden

C. Es existieren keine zuverlässigen Biomarker in der alltäglichen Praxis für Therapieentscheidungen. Therapieentscheidungen sollen deshalb aktuell unter anderem anhand von Vortherapie, Krankheitsaktivität, Funktionsstatus, dem Vorliegen von Erosionen, Sicherheitsaspekten, und der Ko-morbidität sowie der Präferenz des Patienten getroffen werden

D. Der zuständige Arzt für das Management des RA-Patienten, insbesondere für die Aktivitätsbestimmung der RA und Steuerung der medikamentösen Therapie, ist der internistische Rheumatologe

E. Das Ziel der Behandlung ist die Remission (nach dem Prinzip des „treat-to-target"). Niedrigere Krankheitsaktivität kann, wenn nicht anders möglich, eine akzeptable Alternative dazu sein

F. Glukokortikoide sollten bei jedem Patienten ausgeschlichen werden, sofern dies klinisch vertretbar ist. Eine Deeskalation der Basistherapie kann bei Patienten mit einer "sustained remission“ (anhaltende Remission) ohne Glukokortikoidtherapie erwogen werden. Die Deeskalation sollte auf einer gemeinsamen Entscheidung von Arzt und Patient beruhen

\section{Empfehlungen}

1. Sobald die Diagnose einer RA gestellt ist, soll eine Therapie mit DMARD begonnen werden

2. Das Ziel der Therapie ist das Erreichen und die Erhaltung einer Remission

3. Kontrollen der Krankheitsaktivität sollten bei aktiver Erkrankung häufig (alle 1-3 Monate) und mit einem Composite Score erfolgen. Wenn 3 Monate nach Beginn der Therapie keine Verbesserung zu sehen ist oder wenn nach 6 Monaten das Ziel nicht erreicht wird, sollte die Therapie angepasst werden

4. Methotrexat (MTX) soll als erstes csDMARD eingesetzt werden, ein Vorteil für eine initiale Kombination mehrerer csDMARDs ist nicht sicher belegt

5. Falls MTX nicht einsetzbar ist (z. B. wegen Kontraindikationen), soll die Therapie mit Leflunomid oder mit Sulfasalazin begonnen werden

6. Glukokortikoide (GC) sollten bei initialer Therapie ergänzend zum csDMARD gegeben werden. Empfehlenswert ist eine Startdosis bis $30 \mathrm{mg}$ Prednisolonäquivalent/Tag mit Reduktion auf eine niedrige Dosis („low-dose") innerhalb von acht Wochen. Die GC-Therapie soll auf 3-6 Monate beschränkt werden. Eine zusätzliche intraartikuläre GC-Gabe kann sinnvoll sein

7. Bei Verfehlen des Therapieziels mit der optimierten Starttherapie soll die Therapie eskaliert werden. Bei Fehlen von ungünstigen Prognosefaktoren und moderater Krankheitsaktivität kann eine Kombination mehrerer csDMARDs eingesetzt werden. Bei hoher Krankheitsaktivität und/oder Vorliegen ungünstiger Prognosefaktoren soll die Kombination eines csDMARD (in der Regel MTX) mit einem bDMARD oder tsDMARD zum Einsatz kommen

8. Nach unzureichendem Ansprechen zweier csDMARD-Therapien soll eine bDMARD oder tsDMARD-Therapie zum Einsatz kommen 9. Jede bDMARD und tsDMARD-Therapie soll wenn möglich mit MTX kombiniert werden

Empfehlungsgrad

10.

Bei nicht ausreichendem Ansprechen (Verfehlen des Therapieziels) oder Unverträglichkeit der ersten bDMARDTherapie soll der Wechsel auf ein alternatives bDMARD mit gleichem oder anderem Wirkprinzip oder auf ein tsDMARD erfolgen. Ein nochmaliger Wechsel ohne Änderung des Wirkprinzips ist nicht sinnvoll

Wird die Therapie nach csDMARDs mit einem tsDMARD anstatt einem bDMARD begonnen, so sollte bei Nichtansprechen auf ein bDMARD gewechselt werden
A

A

B

A

A

A

A A

D

D

D

D

A

D

A

A

D praktikabler als die, nach den neuen Remissionskriterien ebenfalls mögliche Boole'sche Definition der Remission. In einer Post-Hoc-Analyse der prospektiven randomisierten AGREE-Studie wurde gezeigt, dass Patienten mit einer erreichten SDAI-Remission ein signifikant besseres Outcome sowohl in der Funktion (HAQ) als auch in strukturellen Veränderungen zeigten [100]. In dieser Studie zur Untersuchung der frü- hen RA erreichten ca. $23 \%$ der Patienten bei einer initialen Kombinationstherapie von MTX und einem bDMARD eine SDAI-Remission. Das Ziel der SDAIRemission ist also durchaus realistisch. Wenn nicht anders möglich, kann auch ein Krankheitsaktivitätsscore, der einer niedrigen Krankheitsaktivität entspricht, insbesondere bei schon länger bestehender und schlecht oder unzureichend kontrollierter RA, eine akzeptable Alter- native zum T2T-Ziel Remission sein (zur Definition der Aktivität siehe Glossar, - Tab. 1).

\section{Empfehlung 3}

Kontrollen der Krankheitsaktivität sollten bei aktiver Erkrankung häufig (alle 1-3 Monate) und mit einem Composite Score erfolgen. Wenn 3 Monate nach Beginn der Therapie keine Verbesserung zu sehen ist oder wenn nach 


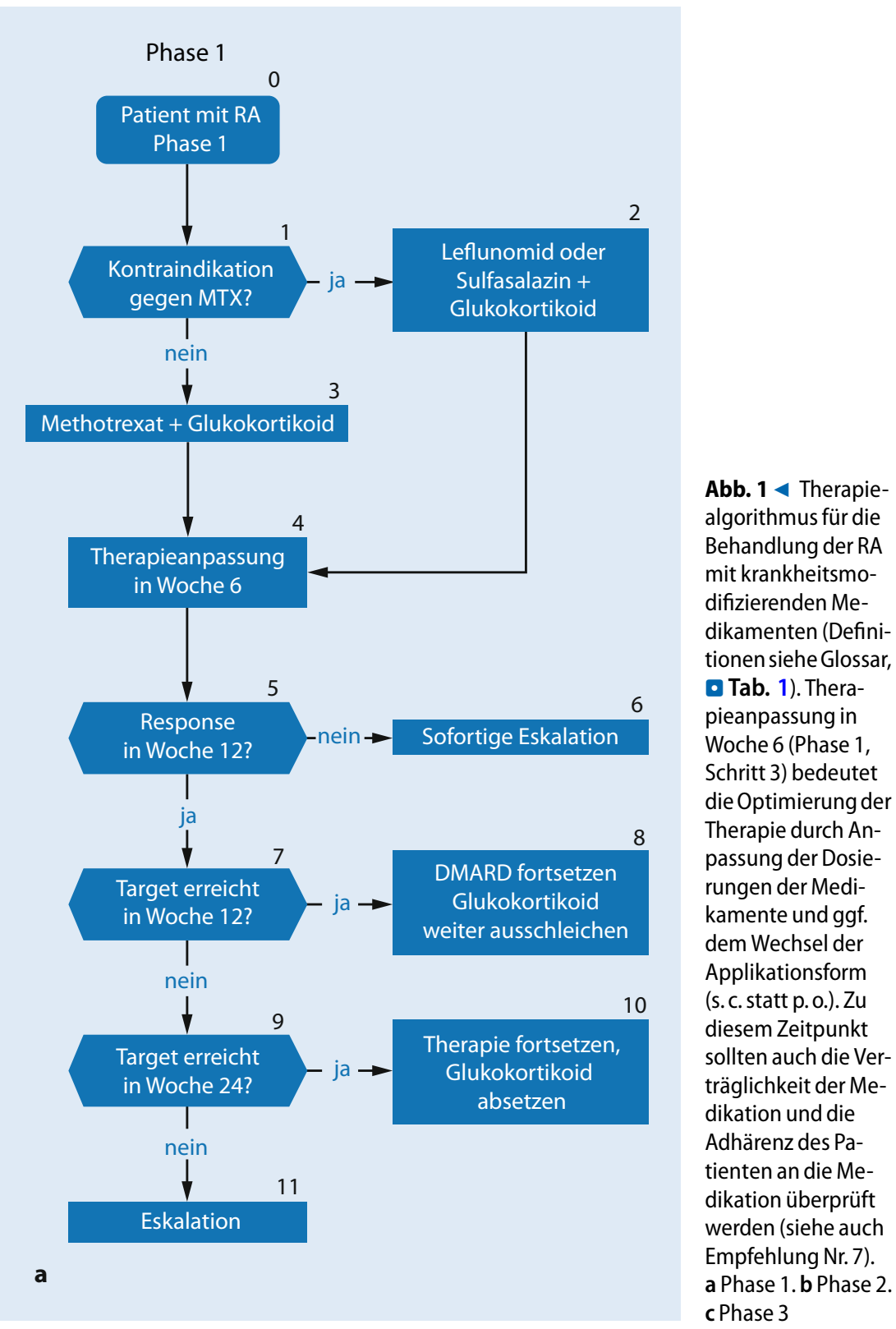

6 Monaten das Ziel nicht erreicht wird, sollte die Therapie geändert werden. (Empfehlungsgrad B)

Konsens war es, sich den Empfehlungen der EULAR anzuschließen [13], dass Kontrollen der Krankheitsaktivität bei aktiver Erkrankung häufig erfolgen sollten (etwa alle 1-3 Monate), mit Änderungen der Therapie nach 3 bzw. 6 Monaten je nach Erreichen einer Verbesserung bzw. des Therapiezieles (-Abb. 1a, Phase 1, Schritt 3-7). Die Verbesserung ist dabei durch eine mindestens $50 \%$ ige Reduktion des jeweiligen Composite Scores definiert (Empfehlungsgrad B). Die Empfehlung ergibt sich aus den Beobachtun-
csDMARDs ist nicht sicher belegt. (Empfehlungsgrad A)

MTX gilt heute in allen Leitlinien unter den konventionellen synthetischen DMARDs (csDMARDs) als Mittel der ersten Wahl („anchor drug“, • Abb. 1a, Phase 1, Schritt 1 [97]). Es ist die innerhalb dieser Gruppe bestuntersuchte Substanz in Monotherapie sowie als Kombinationspartner mit anderen DMARDs. Ein kontrollierter Vergleich mit anderen csDMARDs in der Erstlinientherapie existiert allerdings nicht. Zur Wirksamkeit von MTX in der Monotherapie wurde z.B. in einer Metaanalyse aus sieben RCTs im Dosisbereich 5-25 mg/Woche über 52 Wochen eine im Vergleich $\mathrm{zu}$ Placebo signifikante Wirksamkeit belegt, die gepoolte Risk-Ratio für eine ACR50Response lag bei 3,0, die number needed to treat (NNT) bei 7 [71] (LoE 1a). In der gleichen Auswertung lag die Rate unerwünschter Ereignisse über 12 Wochen unter MTX bei $45 \%$ vs. $15 \%$ unter Placebo, schwere unerwünschte Ereignisse traten über 27-52 Wochen jedoch nicht signifikant häufiger auf (3\% vs. $2 \%$ ). Eine ACR70-Response ist mit einer MTX-Monotherapie innerhalb von 6 Monaten bei $25 \%$ der Patienten erreichbar [91] (LoE 5). Ein optimierter Einsatz in Kombination mit Glukokortikoiden (GC, siehe unten) führt innerhalb von 16 Wochen zu einer Remissionsrate von $73,6 \%$, die auch nach einem Jahr noch bei 60,2\% liegt [122] (LoE 2), wie die randomisierte belgische CareRA-Studie ergab.

In dieser Studie wurde auch gezeigt, dass eine Starttherapie mit mehreren csDMARDs (in diesem Fall MTX + Sulfasalazin bzw. MTX+Leflunomid) keine Vorteile gegenüber MTX + GC bietet und mit einer höheren Rate unerwünschter Wirkungen verbunden ist [122]. Auch in den aktuellen EULAR-Empfehlungen [97] werden nach systematischer Bewertung der Literatur csDMARD-Kombinationen für die Starttherapie nicht mehr empfohlen. Eines der Hauptargumente für diese Entscheidung war neben der besseren Verträglichkeit der Monotherapie, dass in allen Studien, die vermeintlich Vorteile der csDMARD-Kombinationstherapie boten, in der MTX-Kontrollgruppe entweder die Dosierung oder die 
Phase 2

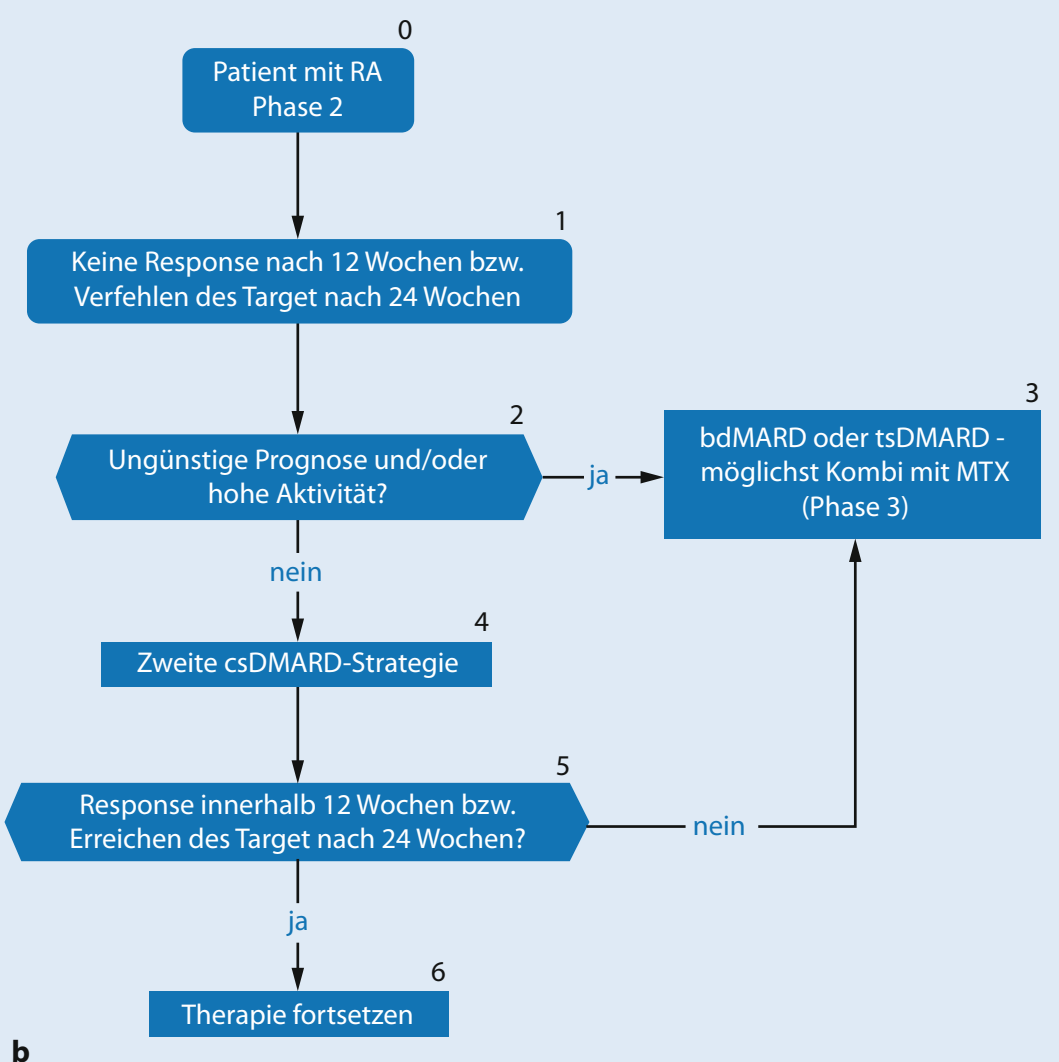

b

Phase 3

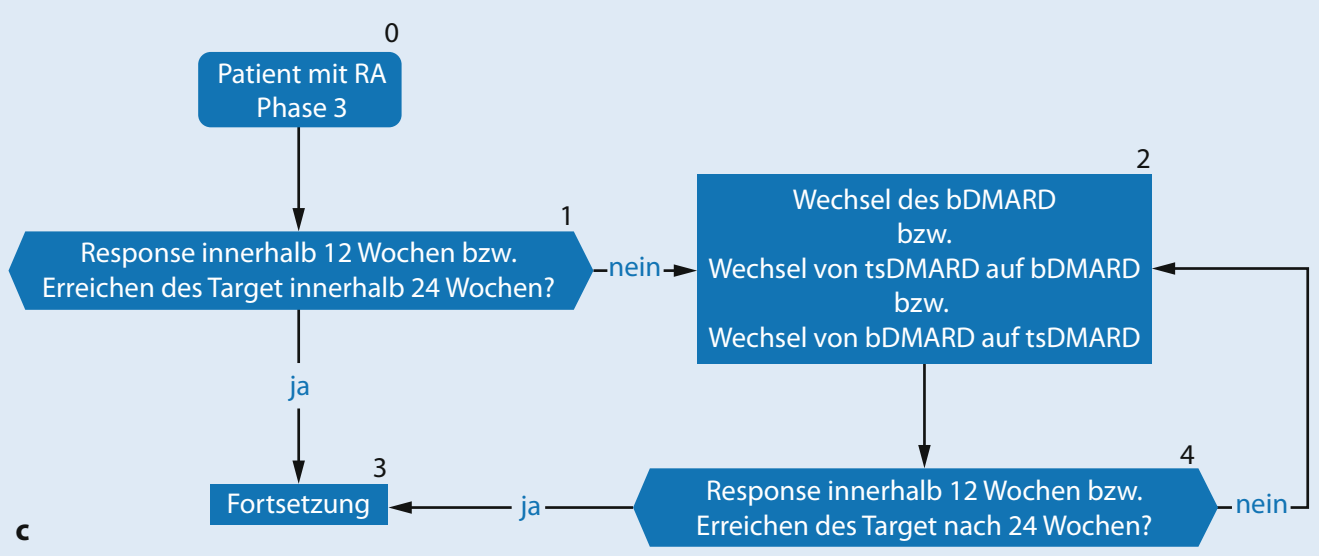

Abb. $1<$ (Fortsetzung)

begleitende GC-Therapie nach heutigem Wissensstand inadäquat gewählt war.

Neben guter Wirksamkeit und Verträglichkeit bietet MTX spezielle Vorteile, wie einen breiten Dosierungsrahmen und verschiedene verfügbare Applikationsformen. Als optimale Startdosis werden bei fehlenden Kontraindika- tionen $15 \mathrm{mg} /$ Woche empfohlen, da hierbei die Balance zwischen möglichst schnellem Wirkeintritt und Verträglichkeit am ausgewogensten scheint [124] (LoE 1a). Eine rasche Dosissteigerung bis auf $25 \mathrm{mg} /$ Woche scheint nach neueren Studiendaten bei fehlenden Gegenanzeigen, wie z.B. Nierenfunktionsstörungen, möglich und erhöht die Wirksamkeit bei nur geringen Verträglichkeitsproblemen: so wurde in der C-EARLYStudie in der MTX-Kontrollgruppe die Dosis zweiwöchentlich um $5 \mathrm{mg}$ bis auf $25 \mathrm{mg}$ gesteigert und damit trotz nichtoptimierter GC-Begleittherapie eine anhaltende Remissionsrate von $15 \%$, LDA- 
Rate von 28,6\% und ACR50-Response von $52,6 \%$ erreicht. Die Rate medikationsbedingter Therapieabbrüche lag bei nur 8,6\% [25] (LoE 1b).

Wegen Resorptionsproblemen bei einem Teil der Patienten und eingeschränkter Bioverfügbarkeit oberhalb $15 \mathrm{mg}$ bei oraler Applikation [87] (LoE 2) erscheint ein Beginn der MTX-Therapie in parenteraler Form (üblicherweise subkutan) vorteilhaft. Das belegt eine RCT mit signifikant höherem ACR20- und ACR70Ansprechen (78 vs. $70 \%$ bzw. 41 vs. $33 \%$ [9]) (LoE 1b) und eine große prospektive kanadische Kohortenstudie [51] (LoE 2), in der die subkutane Applikation u.a. $\mathrm{zu}$ einer signifikant niedrigeren Krankheitsaktivität sowie einer niedrigeren Rate von Therapieversagen und -wechsel führte. Bei erfolglosem Beginn mit einer oralen MTX-Therapie sollte vor einem Substanzwechsel noch ein Versuch mit subkutaner Applikation gemacht werden [76] (LoE 1a).

Die MTX-Gabe sollte standardmäßig durch Folsäure-Substitution (einmalig 5(-10) mg ca. 24h nach der MTX-Gabe) begleitet werden. Nach den Resultaten eines systematischen Reviews [89] (LoE 1a) kann hierdurch das Auftreten von Hepatotoxizität (relative Risikoreduktion $77 \%$ ), gastrointestinalen Nebenwirkungen und Übelkeit (26\%) sowie Abbrüchen wegen schwerer unerwünschter Ereignisse (61\%) signifikant reduziert werden, numerisch auch das Auftreten einer Stomatitis (26\%).

\section{Empfehlung 5}

Falls MTX nicht einsetzbar ist (z. B. wegen Kontraindikationen), soll eine Therapie mit Leflunomid oder mit Sulfasalazin begonnen werden. (Empfehlungsgrad A)

Als therapeutische Alternativen für MTX gelten innerhalb der csDMARDGruppe in erster Linie Leflunomid (übliche Tagesdosis von $20 \mathrm{mg}$ ) oder Sulfasalazin (übliche Tagesdosis $2000 \mathrm{mg}$ ) (• Abb. 1a, Phase 1, Schritt 2). Beide Substanzen haben sich in länger zurückliegenden Studien als ähnlich wirksam wie MTX erwiesen. Allerdings war die MTXDosierung geringer als heute üblich [97]. Für den Vergleich MTX vs. Leflunomid existiert eine Metaanalyse auf der Basis von 4 RCTs, welche die Gleichwertigkeit beider Substanzen belegt [42] (LoE 1a). In einem Placebo-kontrollierten RCT wurden Leflunomid und Sulfasalazin miteinander verglichen und boten ebenfalls gleiche Wirksamkeit [95] (LoE 1b). Antimalariamittel stellen hinsichtlich der Wirkstärke keine gleichwertige Alternative dar, da für sie eine Hemmung der radiologischen Progression nicht nachgewiesen ist [97]. Ihr Einsatz kommt daher - neben der Kombinationstherapie nur in Ausnahmefällen in Frage. Als besonderer Vorteil von Hydroxychloroquin ist jedoch der mehrfach nachgewiesene kardioprotektive Effekt zu erwähnen [88] (LoE 2). Weitere früher häufig verwendete csDMARDs sind heute entweder nicht mehr verfügbar (parenterales Gold) oder kommen nur noch in Ausnahmefällen zur Anwendung (Azathioprin, Ciclospo$\operatorname{rin} \mathrm{A})$.

\section{Empfehlung 6}

Glukokortikoide (GC) sollten bei initialer Therapie ergänzend zum csDMARD gegeben werden. Empfehlenswert ist eine Startdosis bis $30 \mathrm{mg}$ Prednisolonäquivalent/Tag mit Reduktion auf eine niedrige Dosis („lowdose“) innerhalb von acht Wochen. Die GC-Therapie soll auf 3-6 Monate beschränkt werden. Eine zusätzliche intraartikuläre GC-Gabe kann sinnvoll sein. (Empfehlungsgrad A)

GC sind heute aufgrund ihrer unmittelbar einsetzenden anti-inflammatorischen und destruktionsinhibierenden Wirkung ein obligatorischer Bestandteil der Starttherapie in Kombination mit csDMARD (• Abb. 1a, Phase 1). Als optimale Startdosis haben sich in mehreren Studien $30 \mathrm{mg}$ Prednisolon p.o./Tag erwiesen $[20,123]$. Die früher z. B. im COBRA-Schema verwendete höhere Startdosis von $60 \mathrm{mg}$ bietet demgegenüber keinen zusätzlichen Vorteil [20] (LoE 1b). Bisher ist nicht untersucht worden, ob auch Startdosen von 10 oder 20 mg ähnlich gut wie $30 \mathrm{mg}$ wirken (s. ForschungsAgenda). Bis zur Klärung dieser Frage sollte daher die Startdosis fallbezogen im Bereich 10-30 mg gewählt werden. Im weiteren Verlauf sollte die Dosis zügig (möglichst innerhalb von 8 Wochen [97]) reduziert werden mit dem Ziel einer
Tagesdosis von $5 \mathrm{mg}$ Prednisolon oder weniger (siehe $\bullet$ Infobox 1 ).

In einzelnen Studien haben sich weitere initiale GC-Applikationsformen, wie z. B. ein initialer Puls mit $250 \mathrm{mg}$ i. v. Methylprednisolon [78] (LoE 1b) oder die einmalige i. m.-Gabe von $120 \mathrm{mg}$ Methylprednisolon [19] (LoE 1b) als langfristig wirksam erwiesen. Angesichts der gut belegten Risiken von höher dosierten Glukokortikoiden in der Therapie der RA können daraus nach gegenwärtigem Wissensstand aber keine generellen Empfehlungen abgeleitet werden (s. ForschungsAgenda).

Die langfristige Anwendung von GC kann zu unerwünschten Nebenwirkungen führen wie die Osteoporose, als auch kardiovaskuläre Risiken, Infektionsrisiken und gesteigerte Mortalität (Literatur Übersicht unter [82], LoE 1a). Das gilt insbesondere für den Dosisbereich $>5 \mathrm{mg}$ Prednisolon/Tag [106] (LoE 5). Darüber hinaus gibt es keine Evidenz dafür, dass bei optimierter DMARD-Therapie die langfristige GC-Gabe tatsächlich einen zusätzlichen Nutzen mit sich bringt. Der systemische Einsatz von GC sollte deshalb auf einen Zeitraum von 3 bis maximal 6 Monaten beschränkt sein [97]. Die lokal-intraartikuläre Gabe von GC in einzelne Gelenke mit hoher Entzündungsaktivität ist eine in der Praxis viel angewandte zusätzliche Möglichkeit. Es gibt hierzu jedoch nur wenige kontrollierte Kurzzeituntersuchungen und Langzeituntersuchungen fehlen (s. Forschungs-Agenda). In der randomisiert-kontrollierten CIMESTRA-Studie hat diese Therapie als additive Therapie zu ausgezeichneten Langzeitergebnissen in einem untersuchten Zeitraum von bis zu 5 Jahren geführt [53] (LoE 1b).

\section{Empfehlung 7}

Bei Verfehlen des Therapieziels mit der optimierten Starttherapie soll die Therapie eskaliert werden. Bei Fehlen von ungünstigen Prognosefaktoren und moderater Krankheitsaktivität kann eine Kombination mehrerer csDMARDs eingesetzt werden. Bei hoher Krankheitsaktivität und/ oder Vorliegen ungünstiger Prognosefaktoren soll die Kombination eines csDMARD (in der Regel MTX) mit 
Tab. 3 Mögliche Prädiktoren für eine schlechtere Prognose

\begin{tabular}{|c|c|c|}
\hline Prädiktor & Referenz & Evidenzgrad \\
\hline $\begin{array}{l}\text { Nach einer csDMARD-Therapie weiterbestehende, moderate oder } \\
\text { hohe Krankheitsaktivität gemäß akzeptierter Indizes }\end{array}$ & {$[94]$} & $1 b$ \\
\hline Hohe Akute-Phase-Reaktion (z. B. C-reaktives Protein oder BSG) & {$[99,119]$} & $1 b, 2$ \\
\hline Hohe Anzahl geschwollener Gelenke & {$[99,119]$} & $1 b, 2$ \\
\hline Nachweis von Rheumafaktor und/oder Anti-CCP-Antikörper & $\begin{array}{l}{[99,114,} \\
119]\end{array}$ & $1 b, 2,2$ \\
\hline Initial hoher DAS28-Score & [79] & 2 \\
\hline Nachweis von frühen Erosionen & [99] & $1 b$ \\
\hline Versagen von 2 oder mehreren csDMARDs & {$[62]$} & 2 \\
\hline
\end{tabular}

einem bDMARD oder tsDMARD zum Einsatz kommen. (Empfehlungsgrad A)

Wenn nach spätestens 12 Wochen unter optimierter Startherapie noch kein adäquates Therapieansprechen bzw. wenn spätestens nach 24 Wochen noch keine Remission erreicht werden konnte, muss die Therapie angepasst werden (siehe Empfehlung 3 und Abb. 1a, Phase 1, Schritte 3-7). Optimierte Starttherapie in den ersten 6-12 Wochen bedeutet dabei u.a., dass MTX unter Monitoring von Nebenwirkungen und Beachtung von Gegenanzeigen (u.a. Niereninsuffizienz) in der Dosis gesteigert werden sollte (i.R. aber nicht mehr als $25 \mathrm{mg}$ s.c./Woche, siehe Empfehlung 4). Auch die Kontrolle der Adhärenz und der Verträglichkeit der Medikation spielt gerade $\mathrm{zu}$ diesem Zeitpunkt eine wichtige Rolle. Wurde die Therapie mit oralem MTX begonnen, sollte bei fehlendem klinischem Ansprechen binnen 6 Wochen die Therapie auf s.c. umgestellt werden, insbesondere bei Wochendosen $>15 \mathrm{mg}$ (Empfehlung 4, [87]; siehe - Abb. 1a, Phase 1). Auch die kurzfristige Erhöhung der oralen GC-Dosis (i.d.R. nicht $>30 \mathrm{mg} / \mathrm{Tag}$, siehe Empfehlung 6) und/oder intraartikuläre GC-Injektionen in einzelne Gelenke können in der ersten Phase nötig sein. Die weitere Therapieentscheidung sollte dann anhand von Prognosefaktoren erfolgen (siehe - Tab. 3; - Abb. 1b, Phase 2, Schritt 2). Fehlen negative Prognosefaktoren, kann zu Woche 12 bzw. 24 eine Änderung der CsDMARD-Strategie erwogen werden (• Abb. 1b, Phase 2, Schritt 4). Eine Kombinationstherapie verschiedener csDMARD-Therapien, die MTX beinhaltet, ist in dieser Situation einem Wechsel auf eine MTX-freie csDMARD-Therapie vorzuziehen [63]. Die robusteste Datenlage besteht für eine Kombinationstherapie verschiedener csDMARDs nach dem s.g. O'Dell-Schema, bestehend aus MTX/Sulfasalazin/Hydroxychloroquin [50], wobei es keinen direkten Vergleich gegen die auch häufig angewandte Kombination aus MTX/Leflunomid gibt. Im Falle einer frühen MTX-Intoleranz sollte eine alternative csDMARD-Therapie gewählt werden.

Beim Vorliegen ungünstiger Prognosefaktoren (siehe -Tab. 3) sollte bei Verfehlen des Therapiezieles unter optimierter Starttherapie (ausreichendes Ansprechen zu Woche 12 bzw. Remission $\mathrm{zu}$ Woche 24) bereits früher eine Therapie mit einem bDMARD oder einem tsDMARD erwogen werden (siehe - Abb. 1b, Phase 2, Stufe 3). Eine Präferenz für die eine oder andere Substanzgruppe kann an dieser Stelle nicht ausgesprochen werden (siehe Empfehlung 8). Auf Grund der längeren Erfahrung kommen bisher nach dem ersten csDMARD-Versagen bevorzugt bDMARDs zur Anwendung. Alle bDMARDs oder tsDMARDs sollten, sofern möglich mit MTX kombiniert werden (siehe auch Empfehlung 9).

\section{Empfehlung 8}

Nach unzureichendem Ansprechen zweier csDMARD-Therapien soll eine bDMARD- oder tsDMARD-Therapie zum Einsatz kommen. (Empfehlungs$\operatorname{grad}$ A)

Der sequentielle Einsatz mehrerer csDMARDs bzw. csDMARD-Kombinationen führt nach den Ergebnissen der BeST-Studie und aus der britischen ERAN-Kohorte spätestens ab dem 2. Wechsel nur noch $\mathrm{zu}$ einem sehr geringen Zuwachs an Respondern. Bei Verzicht auf eine Eskalation in Form von zielgerichteten Therapien mit bDMARD oder tsDMARD kommt es in einem großen Anteil zum Nichterreichen der Therapieziele [41, 62] (LoE 2). Darüber hinaus ergibt sich mit der Anzahl der erfolglosen Wechsel ein beträchtlicher Zeitverlust, der eine vermehrte radiologische Destruktion [41] und dauerhaft einen eingeschränkten Funktionsstatus [62] mit sich bringt.

Hieraus ergibt sich, dass bDMARDs bzw. tsDMARDs bei unzureichendem Ansprechen zweier csDMARD-Therapien spätestens 6 Monate nach Behandlungsbeginn zum Einsatz kommen sollten (-Abb. 1, Phase 2, Stufe 3). Welches bDMARD oder tsDMARD verwendet wird, kann in Anbetracht der Gleichwertigkeit der zur Verfügung stehenden Substanzen individuell (z. B. anhand patientenindividueller Kriterien) entschieden werden (s. Forschungs-Agenda). Rituximab hat zwar erst nach dem Versagen mindestens eines TNF-Inhibitors eine Zulassung, in besonderen Therapiesituationen (z. B. Lymphom- oder TBCAnamnese, begleitende Vaskulitis o.ä.) kann aber der Einsatz auch schon direkt nach Versagen von csDMARDs sinnvoll sein (LoE 5).

tsDMARDs, derzeit namentlich die JAK-Inhibitoren (JAKi) Tofacitinib und Baricitinib, sind eine gleichwertige alternative Therapieoption zu bDMARDs nach Versagen einer csDMARD-Therapie (LoE 1b; - Abb. 1b, Phase 2, Schritt 3; $[23,34,64,108,113,120])$. In einer randomisierten Studie wurde eine Überlegenheit von Baricitinib gegenüber Adalimumab jeweils in Kombination mit MTX bezüglich des primären Endpunktes (ACR20-Ansprechen) gezeigt [108]. Die gegenwärtige Studienlage erlaubt allerdings nicht, eine generelle Überlegenheit bezüglich der Wirksamkeit der JAKi gegenüber der gesamten Klasse der bDMARDs anzunehmen (LoE 5).

\section{Empfehlung 9}

Jede bDMARD- und tsDMARD-Therapie soll wenn möglich mit MTX kombiniert werden. (Empfehlungsgrad A)

Für alle bDMARDs und tsDMARDs liegen Studienergebnisse vor, die Vor- 


\section{Infobox 1 \\ "Niedrig-dosierte" Glukokor- tikoidtherapie und "angestrebte" Glukokortikoiddosis bei RA}

Die noch gültige Definition der EULAR für eine niedrig-dosierte Glukokortikoidtherapie als Langzeittherapie liegt bei $\leq 7,5 \mathrm{mg}$ Prednisolonäquivalent/d ([24, 54]; siehe Glossar, - Tab. 1 und Empfehlung 6). Dies beinhaltet das mögliche Missverständnis, dass es sich hierbei um eine Dosis mit vertretbaren Risiken handelt. Tatsächlich wurden in den letzten Jahren eine Vielzahl von Daten erhoben, welche zeigen, dass Glukokortikoide dosisabhängig auch im niedrigen Dosisbereich signifikante Risiken, z. B. durch ein erhöhtes Infektionsrisiko [68], mit sich bringen. Eine Arbeitsgruppe der EULAR hat dies systematisch untersucht und ist zu der Meinung gekommen, dass nur in einem Dosisbereich von $\leq 5 \mathrm{mg}$ Prednisolonäquivalent ein akzeptabel niedriges Risiko für eine Gefährdung des Patienten durch die Therapie besteht [106]. Dies auch nur bei Patienten, welche kein hohes Risiko für eine kardiovaskuläre Erkrankung haben. Analog zu den "Klug entscheiden"-Empfehlungen der DGRh von 2016 [32] empfiehlt die Konsensgruppe daher, dass eine Dosis von $5 \mathrm{mg}$ Prednisolonäquivalent als Langzeittherapie nicht überschritten werden sollte. Die Glukokkortikoidtherapie sollte auf die niedrigst-mögliche Dosis, am besten bis zum kompletten Absetzen, reduziert werden. Bezüglich der idealen Glukokortikoiddosis zum Therapiebeginn (Empfehlung 6) soll betont werden, dass eine Dosis bis $30 \mathrm{mg}$ Prednison/d bedeutet, dass diese i. $d$. $R$ nicht überschritten werden sollte und häufig auch niedrigere Dosen indiziert und ausreichend sind.

Die jeweils gültigen Leitlinien-basierten Empfehlungen zur Prophylaxe der Osteoporose, wie z. B. die Substitution von Vitamin D und ggf. Calcium sollten beachtet werden.

teile für die Kombination mit MTX im Vergleich zu monotherapeutischem Einsatz zeigen (Übersicht unter [15, 77], LoE 1a). MTX sollte daher bei fehlenden Kontraindikationen zumindest so lange kombiniert werden, bis eine anhaltende Remission vorliegt. MTX kann in dieser Kombination niedriger dosiert werden als bei alleinigem Einsatz von MTX. Unter randomisiert-kontrollierten Bedingungen erwies sich eine wöchentliche MTX-Dosis von $10 \mathrm{mg}$ im Vergleich zu
$20 \mathrm{mg}$ als Kombinationspartner von Adalimumab als gleichwertig [11] (LoE 1b).

Steht MTX als Kombinationspartner nicht zur Verfügung, so liegen für die IL6-Rezeptor-Inhibitoren Tocilizumab und Sarilumab als auch für das tsDMARD Baricitinib die besten Monotherapie-Ergebnisse vor: Tocilizumab hat in zahlreichen Studien eine gute monotherapeutische Wirkung bewiesen (Übersicht unter $[16,77])$ und sich ebenso wie Sarilumab im Head-to-Head-Vergleich mit Adalimumab als monotherapeutisch überlegen erwiesen [12, 37] (LoE 1b). Baricitinib zeigt klinisch in der Monotherapie die gleiche Wirksamkeit wie in Kombination mit MTX, die Kombination bietet jedoch im radiologischen Outcome noch Vorteile [36] (Evidenzgrad 1). Im Fall von Tofacitinib zeigt eine neue, erst nach der systematischen Literaturrecherche publizierte Vergleichsstudie mit Adalimumab, dass Tofacitinib nur in Kombination mit MTX, nicht aber als Monotherapie, gleichwertig zu Adalimumab ebenfalls in Kombination mit MTX ist [35]. JAKi sollten deswegen mit csDMARDs kombiniert werden (Evidenzgrad 5). Für die Monotherapie besteht in Deutschland eine Zulassung für Baricitinib, für Tofacitinib nur, wenn MTX nicht vertragen wird oder eine Therapie mit MTX ungeeignet ist $[36,66]$.

Für die Anwendung anderer csDMARDs als Kombinationspartner für bDMARDs oder tsDMARDs ist die Evidenz bisher sehr begrenzt: Nichtinterventionelle und Kohortenstudien zeigen (bei teils kleinen Fallzahlen), dass auch Leflunomid als Kombinationspartner geeignet ist, liefern aber auch widersprüchliche Ergebnisse [73] (LoE 2) (s. Forschungs-Agenda).

\section{Empfehlung 10}

Bei nicht ausreichendem Ansprechen (Verfehlen des Therapieziels) oder Unverträglichkeit der ersten bDMARDTherapie soll der Wechsel auf ein alternatives bDMARD mit gleichem oder anderem Wirkprinzip oder auf ein tsDMARD erfolgen. Ein nochmaliger Wechsel ohne Änderung des Wirkprinzips ist nicht sinnvoll. (Empfehlungsgrad A) Wird die Therapie nach csDMARDs mit einem tsDMARD anstatt einem bDMARD begonnen, so sollte bei Nichtansprechen auf ein bDMARD gewechselt werden. (Empfehlungsgrad $D$ )

Wenn sich nach Versagen von csDMARDs auch eine bDMARD-Therapie als nicht ausreichend wirksam erweist, so ist prinzipiell der Wechsel auf ein weiteres bDMARD mit gleichem Wirkmechanismus, auf ein bDMARD mit anderem Wirkmechanismus oder auf ein tsDMARD möglich (- Abb. 1c, Phase 3, Schritt 2). Gute Ergebnisse sind in kontrollierten Studien für alle 3 Möglichkeiten in RCTs, Register-Auswertungen und Metaanalysen gezeigt worden, formell allerdings nur nach Versagen einer TNF-Inhibitor-Therapie $[10,38,47,48,93,96]$ ( $\operatorname{LoE} 1 b)$. Eine bessere Wirkung bei Wechsel des Wirkmechanismus nach vorheriger erfolgloser anti-TNF-Therapie wird durch eine randomisierte Studie mit offenem Design nahegelegt [43] (LoE 2). Aus dieser methodisch nicht unproblematischen Studie kann aber eine generelle Empfehlung für einen solchen Wechsel noch nicht abgeleitet werden (s. ForschungsAgenda).

Für den Einsatz von JAKi nach Versagen von nicht-TNF-Biologika liegen keine ausreichenden Studiendaten vor, es ist jedoch anzunehmen, dass auch dieses Vorgehen möglich ist ( $\mathrm{LoE} 5$ ).

Eine evidenzbasierte Empfehlung für das weitere Vorgehen, nachdem ein tsDMARD kein ausreichendes Ansprechen gezeigt hat, kann bisher nicht gegeben werden, da weder für den nachfolgenden Wechsel auf ein bDMARD noch auf ein zweites tsDMARD Daten aus Studien oder ausreichende Erfahrungen vorliegen (s. Forschungs-Agenda).

Es fehlen derzeit noch randomisierte Placebo-kontrollierte Studien für die Wirksamkeit und Sicherheit von 1.) antiTNF-Therapie nach bDMARD mit anderen Wirkprinzipien, 2.) anti-IL-6-Therapie nach anderen anti-IL-6-Therapien oder 3.) JAKi-Therapie nach einer Therapie mit einem nicht-anti-TNF bDMARD oder einem anderen JAKi (s. ForschungsAgenda).

Biosimilars. Die Konsensgruppe stimmt mit der kürzlich veröffentlichten Emp- 
fehlung einer internationalen Task Force überein [60] (LoE 5), dass zugelassene Biosimilars für bDMARDs in gleicher Weise wie die entsprechenden Originalpräparate eingesetzt werden können, um die jeweils geeigneten Patienten zu behandeln.

Bei nicht ausreichender Wirksamkeit eines Originator-Biologikums (boDMARD) wird ein Wechsel zum korrespondierenden Biosimilar (bsDMARD) nicht empfohlen, auch wenn formell ein solches Vorgehen nicht untersucht ist (Empfehlungsgrad D).

\section{Treat-to-target (T2T)}

\section{Nutzen von T2T}

Der Nutzen von T2T in der Behandlung der RA wurde in einer Vielzahl von Studien, (als Beispiel angeführt die NEORACo-Studie [81]) immer wieder gut belegt. Die aktuelle Datenlage dazu haben Stoffer et al. [101] zusammengefasst. Es gibt daher einen Konsens, dass T2T in der Behandlung der RA wichtig und empfehlenswert ist (Empfehlungsgrad A, siehe übergeordnete Empfehlung E). Dies sollte konsequent geschehen (Empfehlungsgrad B), denn eine geringe Adhärenz des Arztes zu T2T verschlechtert den Outcome des Patienten [64]. Die Herausforderung des T2T ist daher vor allem, dieses auch tatsächlich in der Praxis zu implementieren. Systematische Untersuchungen der Umsetzung von T2T in der Versorgungswirklichkeit, wie z. B. die CAPEA-Studie [1], und auch regelmäßige Aufklärung nicht nur von Rheumatologen, sondern auch rheumatologischen Fachassistenten und Patienten über die Wichtigkeit von T2T sind notwendig, damit T2T besser wirksam werden kann.

\section{Messmethoden und Ziele des T2T Anwendung von T2T in speziellen Po- pulationen von RA-Patienten. Die Kon-} sensgruppe hat die Frage untersucht, ob es Populationen innerhalb der Patienten mit RA gibt, für die T2T nicht anzuwenden ist. Studien zur Anwendung von T2T bei älteren RA-Patienten, bei Menschen mit Komorbidität/en oder mit länger existierender RA liegen jedoch nicht vor. Die Evidenz ist daher als gering $\mathrm{zu}$ bewerten. Es gibt aber andererseits keine Hinweise, dass bei diesen Populationen T2 $\mathrm{T}$ nicht angewendet werden kann (s. Forschungs-Agenda). Da es Daten aus internationalen Registern und der deutschen Kerndokumentation gibt [56], dass ältere Patienten mit RA oft inadäquat behandelt werden, sieht die Konsensgruppe auch hier die Notwendigkeit der Anwendung von T2T (Empfehlungsgrad D). Die Meinung der Konsensgruppe ist, dass Alter und Komorbidität kein Grund sind, auf das Prinzip des T2T zu verzichten. Individuelle Faktoren müssen allerdings berücksichtigt werden.

Bildgebung bei T2T. Die Messung der strukturellen Veränderungen im konventionellen Röntgen mit Hilfe von validierten Scores [111] ist der Goldstandard zur Messung des Verlaufs der RA. Bewertet werden dabei die Erosivität und die Gelenkspaltverschmälerung der Gelenke von Händen und Füßen. Neue Therapien der RA haben alle an dem Kriterium der Hemmung der radiologischen Progression ihre Wirkung belegt. Unter dem Einfluss moderner Therapiestrategien ist die Anzahl der Patienten mit radiologisch sichtbaren Strukturveränderungen jedoch deutlich geringer geworden und T2T hat das Ziel, dessen Auftreten primär zu verhindern. Als empfohlene Methode des T2T werden die radiologischen Kontrolluntersuchungen in der hier vorgestellten Leitlinie deswegen nicht mehr erwähnt. Unabhängig spielt die Röntgenuntersuchung und insbesondere der Nachweis von Erosionen im konventionellen Röntgen aber immer noch eine wichtige Rolle, sowohl für die initiale Diagnosestellung der RA, für die Prognoseabschätzung, wie auch im Falle eines Neuauftretens oder einer Zunahme von Erosionen als Signal zur Therapieanpassung.

Zur Bedeutung von Ultraschall im Management der RA gibt es eine Vielzahl von aktuellen Arbeiten. So zeigen prospektive Kohortenstudien, dass die Nutzung von Ultraschall zu einer besseren Einschätzung der Krankheitsaktivität bei RA-Patienten führen kann [18, 74] und dass Ultraschall sehr rasch ein Ansprechen auf eine Therapie mit einem bDMARD nachweist [74]. Zwei aktuell publizierte, prospektive randomisierte Studien zur frühen RA, die ARTIC- und die TaSER-Studie $[18,46]$, haben untersucht, ob der Einschluss von validierten Ultraschall Scores in einen klinischen Composite Score im Rahmen des T2T $\mathrm{zu}$ einem besseren Outcome im Vergleich zu einer Gruppe ohne Ultraschall führt. Das Ergebnis war jedoch, dass das Zielkriterium der Remission in beiden Studien mit oder ohne Nutzung von Ultraschall genauso häufig erreicht wurde. In der TaSER-Studie zeigte die Ultraschallgruppe außerdem einen häufigeren Einsatz von bDMARDs, ohne dass ein signifikanter klinischer Nutzen eintrat.

Nach der derzeitigen Studienlage zur frühen RA verbessert eine Therapiestrategie, die sich an einer sonographischen Remission orientiert, verglichen mit einer DAS28-orientierten Strategie das mittelfristige Outcome der Patienten nicht, trotz des vermehrten Einsatzes intensiver und teurer Therapien in dieser Gruppe. Ein Langzeit-Follow-up dieser Studien existiert jedoch noch nicht (s. Forschungs-Agenda). Die Benutzung eines Composite Scores mit einem Ultraschall unter Einbezug der Gelenke, kann im Rahmen des T2T daher bisher nicht empfohlen werden (Empfehlungs$\operatorname{grad}$ A).

Die Datenlage zum Einsatz von MRTKontrollen im T2T ist bisher für eine Beurteilung noch nicht ausreichend. Da$\mathrm{zu}$ läuft derzeit eine prospektive randomisierte Studie (www.clinicaltrials.gov; IMAGINE-RA NCT01656278).

Es ist aufgrund o.g. Daten Konsens in der Leitliniengruppe, dass Ultraschall und MRT wichtige Instrumente für die Beurteilung der Krankheitsaktivität, hilfreich bei der Diagnosestellung und in Einzelfällen auch für Therapieentscheidungen der RA sind. Ihr genereller Nutzen für die Therapieentscheidung nach T2T der RA ist jedoch noch nicht ausreichend belegt (s. Forschungs-Agenda).

\section{Biomarker und individualisierte Medizin}

Das Thema „individualisierte Medizin“ (engl.: personalized health care (PHC)) hat in den letzten Jahren, u. a. aufgrund der zahlreichen neuen zur Verfügung ste- 


\section{Infobox 2 \\ Lebensstil-Interventionen und DMARD-Therapie bei RA}

Es existiert ein gut belegter Zusammenhang sowohl zwischen Adipositas [72] wie auch Rauchen $[85,86]$ mit einer schlechteren Prognose wie auch geringerem Ansprechen auf DMARD-Therapien bei RA. Darüber hinaus erhöhen diese Faktoren das kardiovaskuläre Risiko und haben negativen Einfluss auch auf andere Komorbiditäten. Die Einwirkung auf eine Lebensstiländerung gehört daher bei betroffenen RA-Patienten zu den ärztlichen Aufgaben. Ob eine Intervention mit dem Ziel der Gewichtabnahme und der Rauchentwöhnung den Verlauf und das Ansprechen auf DMARDs jedoch verbessern kann, ist noch nicht abschließend geklärt. Bei einer prospektiven Untersuchung mit übergewichtigen RA-Patienten und stabiler DMARD-Therapie zeigte eine Reduktionsdiät eine Verbesserung der Krankheitsaktivität abhängig vom Ausmaß der Gewichtabnahme [45]. Auch nach bariatrischer Chirurgie bei RA-Patienten wurde eine deutliche Verbesserung der Krankheitsaktivität beobachtet [102]. Im Gegensatz dazu war in einer Kohortenuntersuchung das Beenden des Rauchens im Beobachtungszeitraum von 2-8 Jahren nicht mit einem günstigeren Verlauf assoziiert, es zeigte sich sogar eine etwas höhere Krankheitsaktivität in dieser Gruppe [7]. Prospektive, kontrollierte Studien müssen daher die Wirksamkeit von Lebensstilinterventionen auf das DMARD-Ansprechen in Zukunft noch besser belegen. Wegen des großen Nutzens auf die Komorbiditäten der RA werden das Anstreben eines normalen BMI wie auch die Rauchentwöhnung jedoch trotzdem stark empfohlen.

henden medikamentösen Interventionsmöglichkeiten, weiter an Bedeutung gewonnen. Die Leitliniengruppe der DGRh hält daher - analog zur EULAR - zu diesem Thema eine übergeordnete Empfehlung für sinnvoll (• Tab. 2, C). Es bestand eine große Übereinstimmung, dass das Konzept einer personalisierten Medizin mittels Biomarkern sinnvoll ist. Biomarker sollten u.a. die Auswahl bestimmter Medikamente für bestimmte Patientengruppen erleichtern, das Ansprechen auf die Therapien oder auch für $\mathrm{Ne}$ benwirkungen voraussagen und eine Individualisierung in der Dosierung oder der Beendigung der Therapien ermöglichen. Hierzu wurden in den letzten Jahren eine Reihe von interessanten und vielversprechenden Studien und Ansätzen publiziert. Allerdings gibt es bisher keine in der alltäglichen Praxis etablierte und anwendbare Biomarker, die eine Individualisierung auf Patientenebene erlauben (s. Forschungs-Agenda). Die umfangreichste Datenlage existiert aktuell zu der Frage, ob Anti-CCP-Antikörper und/ oder Rheumafaktoren (RF) Prädiktoren für Ansprechen sind. Es gibt Hinweise, dass beide Zell-gerichteten Therapien (anti-CD20 und CTLA-4-Ig) im Falle eines hoch positiven Antikörpernachweises (Anti-CCP) in Bezug auf Abatacept und eines positiven Antikörpernachweises (Anti-CCP und RF) in Bezug auf Rituximab eine Überlegenheit für das klinische Ansprechen (z.B. DAS28-Reduktion oder Erreichen der Remission) gegenüber einer TNF-Inhibitor-Therapie haben könnten [27, 44, 57, 101]. Die Evidenzstärke ist aber nicht so hoch, dass eine generelle Empfehlung für oder gegen eine bestimmte Therapie bei AntiCCP-/RF-positiven Patienten ausgesprochen werden kann (Evidenzstärke C).

Weitgehender Konsens besteht darin, dass Patienten mit Autoantikörpern i.S. von Anti-CCP-Antikörpern und/oder RF (insbesondere bei hohen Konzentrationen) eine schlechtere Prognose für den Verlauf der RA und gewisser Ko-morbiditäten haben. Diese Tatsache bildet sich auch in dem konsentierten Therapiealgorithmus zusammen mit früher Erosivität als ungünstiger Prognosefaktor ab (siehe - Abb. 1b). Andere mögliche Prädiktoren für eine schlechtere Prognose sind in - Tab. 3 aufgeführt.

Weitere Beispiele von Prädiktoren für ein schlechteres Ansprechen sind u.a. auch folgende Konstellationen:

Höheres Alter der Patienten [80] und längere Krankheitsdauer der RA, inkomplette B-Zell-Depletion bei einer RTX-Therapie [125], der Nachweis von s.g. anti-drug-Antikörpern (ADA) bzw. niedrige Medikamentenspiegel im Serum ([75], s. unten), Rauchen (siehe auch - Infobox 2) sowie das initiale Nichtansprechen auf GC, bzw. eine lange Dauer bis zum ersten Ansprechen unter DMARDs [127].

Eine besondere Situation besteht für Patienten, bei denen wegen einer Kontraindikation oder einer Nebenwirkung kein MTX in Kombination mit einem bDMARD oder tsDMARD gegeben werden kann. Für diese Patienten hat sich ein Vorteil einer IL-6-Rezeptor- oder einerJAK-Inhibition mit Baricitinib gegenüber einem TNF-Inhibitor gezeigt (jeweils in Studien gegen Adalimumab, siehe auch Therapiealgorithmus - Abb. 1b, Phase 2, Schritt 3; [12, 13, 22, 36, 37, 59]). Insgesamt ist der Einsatz von MTX bei guter Verträglichkeit in der Regel einer Monotherapie mit bDMARDs weiter vorzuziehen (siehe Empfehlung 9), u.a. auch, weil die Induktion von ADA reduziert und dadurch das Risiko eines sekundären Wirkverlustes gesenkt werden kann. Trotz der Hinweise, dass der Nachweis von Antikörpern gegen bDMARDs und/oder niedrige Serum-Konzentrationen mit einem schlechteren Ansprechen bzw. mit einem Wirkverlust assoziiert sein können, wird die routinemäßige Bestimmung von ADA zur Steuerung der Therapie gegenwärtig noch nicht empfohlen.

Neben Prädiktoren für ein gutes bzw. fehlendes Ansprechen besteht auch Bedarf an Prädiktoren für unerwünschte Ereignisse, um durch die gezieltere Auswahl von DMARDs bei Patienten potentielle Nebenwirkungen zu vermeiden. Auch hier existiert kein singulärer Marker, der im Rahmen der Leitlinie empfohlen werden kann (s. ForschungsAgenda). Ein erhöhtes Infektionsrisiko besteht aber insbesondere in den ersten 6 Monaten nach Beginn einer bDMARDTherapie. Generelle Risikofaktoren für Infektionen stellen außerdem u. a. vorausgegangene Infekte, die Anzahl der csund bDMARD-Vortherapien, ein hohes Alter, GC in höheren Dosierungen, Komorbiditäten wie Lungenerkrankungen oder Niereninsuffizienz oder eine hohe Krankheitsaktivität mit konsekutiver Immobilisierung (z.B. reduziertem HAQ) dar $[68,105,129]$. Das individuelle Risiko für Infektionen kann unter http://www. biologika-register.de/home/risikoscorefuer-infektionen/ abgeschätzt werden.

Zusammenfassend muss jede Substanz sorgsam anhand von Sicherheitsaspekten und den Komorbiditäten des Patienten ausgewählt werden. Für eine Empfehlung bestimmter Substanzen in individuellen Therapiesituationen fehlt 


\section{Infobox 3 \\ Partizipative Entscheidungs- findung (PEF, engl. shared decision-making)}

Die Empfehlung, die Behandlung der Patienten auf der Basis einer gemeinsamen Entscheidungsfindung durchzuführen, findet sich heute in den übergeordneten Prinzipien wichtiger Leitlinien zur Therapie der RA [90, 97] ebenso wie in den Empfehlungen der internationalen T2T-Task Force [92]. Gemeint ist damit eine umfassende Besprechung, die u. a. Informationen über Krankheitsmerkmale, diagnostische Prinzipien, Therapieziele und Nutzen/Risiko der einzelnen Therapieoptionen beinhaltet und auf dieser Basis in gemeinsame Entscheidungen mündet. Als Richtschnur hierfür kann eine Sammlung von 16 "Standards of Care" gelten, die von einer europäischen Arbeitsgruppe (eumusc.net project) unter Mitwirken von Patienten erarbeitet wurde [104].

Die PEF stärkt die Zufriedenheit des Patienten und kann zu einer Optimierung der Beziehung zwischen Arzt und Patient führen. Daneben ist mehrfach nachgewiesen worden, dass die Adhärenz gegenüber der medikamentösen Therapie hierdurch verbessert wird $[70,81]$. Noch unklar ist, inwieweit sich auch das Langzeit-Outcome der RA damit verbessern lässt. Als Argument gegen die Machbarkeit der partizipativen Entscheidungsfindung wird oft der Zeitmangel in der rheumatologischen Sprechstunde angeführt. Hier gilt es sicherlich Kompromisse zwischen Zeitablauf und Patientenwünschen zu schließen. Abhilfe könnte z.B. die Unterstützung im Rahmen der Patientenedukation durch geschulte Fachassistenz schaffen. Die häufige Konstellation, in welcher Patienten den Wunsch äußern, die Therapieentscheidungen dem Rheumatologen zu überlassen, kann als Sonderform der PEF akzeptiert werden. Das Prinzip der PEF gilt für sämtliche Empfehlungen dieser Leitlinie, von besonderer Bedeutung ist PEF jedoch als Grundlage eines Therapieabbaus im Stadium der anhaltenden Remission (siehe Kap. Deeskalation) und des Wechsels vom boDMARD zum bsDMARD. Wenn Kostenfaktoren Therapieentscheidungen bestimmen, wie $z$. B. bei der generellen Umsetzung von bDMARDs auf bsDMARDs in manchen Gesundheitssystemen [40] oder bei Einflussnahme von Kostenträgern auf medizinische Entscheidungen, werden die Prinzipien des PEF oft nicht berücksichtigt. Dies ist auch einer der wichtigen Kritikpunkte an solchen Maßnahmen. generell eine hohe Evidenz und es wird deshalb im Rahmen dieser Leitlinie auf konkrete Empfehlungen verzichtet. In der individualisierten Medizin im Sinne einer "PHC“ liegt ohne Zweifel ein möglicher Schlüssel für ein besseres Outcome und eine Reduktion von Nebenwirkungen und ggf. auch von Kosten. Der momentane Therapiealltag muss aber noch ohne solche „Biomarker" auskommen und es besteht hoher Bedarf an Studien und wissenschaftlichen Projekten, die in der ForschungsAgenda aufgeführt sind.

\section{Deeskalation der DMARD-Therapie}

\section{Option der Deeskalation}

Die Autoren unterscheiden zwischen der Deeskalation von GC, die auf jeden Fall weitmöglichst erfolgen sollte, und der von DMARDs (siehe übergeordnetes Prinzip F, - Tab. 2). In der Regel sollten zunächst die GC beendet werden, bevor überhaupt eine Deeskalation des/der DMARDs in Erwägung gezogen wird. Eine Deeskalation der DMARD-Therapie kann unter bestimmten Bedingungen sinnvoll sein (siehe unten). Nach derzeitigem Kenntnisstand existieren außer unerwünschten Wirkungen keine konkreten medizinischen Gründe, die eine Deeskalation notwendig machen würden, insbesondere da der Krankheitsverlauf unter reduzierter Therapie nicht mit hinreichender Wahrscheinlichkeit vorhergesagt werden kann. Medizinisch ist daher eine Abwägung von potentiellem Nutzen durch temporäre Vermeidung einer Exposition von Medikamenten gegenüber dem Risiko eines Schubes („Flare“; Definition siehe Glossar, • Tab. 1) nicht möglich. Im Falle einer langfristig erfolgreichen Deeskalation kann diese von gesundheitsökonomischem Vorteil sein. Eine finale Einschätzung der Kosteneffizienz einer Deeskalation ist schwierig, da es bisher keinen anerkannten Schwellenwert der Akzeptanz gibt, wie viele Jahre Lebensqualität man bereit ist zu verlieren, um im Gegenzug Kosten einzusparen.

\section{Kriterien für eine Deeskalation} Wenn die Therapie deeskaliert werden soll, muss nach erfolgtem Ausschlei- chen der Glukokortikoidmedikation (vgl. Empfehlung 6) eine anhaltende Remission („sustained remission“) über mindestens 6 Monate bestehen (Empfehlungsgrad D).

Grundsätzlich muss das Ziel sein, unter einer Deeskalation die Krankheitskontrolle zu erhalten. Nach welchen Kriterien ein Patient für eine Deeskalation selektiert werden kann, ist aufgrund der aktuellen Evidenzlage nicht zu beantworten. Wichtig - wie bei allen Therapieentscheidungen - ist es, dass eine Deeskalation nur nach den Grundsätzen der partizipativen Entscheidungsfindung (engl.: „shared decision" siehe übergeordnetes Prinzip A und - Infobox 3) erfolgt.

In den bisher vorliegenden Deeskalationsstudien wurden verschiedene Kriterien für eine Remission oder niedrige Krankheitsaktivität herangezogen, die als Voraussetzung für eine Deeskalation dienten.

In der Regel wird in diesen Studien eine sog. anhaltende Remission („sustained remission") als Einschlusskriterium gefordert. Diese ist üblicherweise über einen DAS28-Score <2,6 über mindestens 6 Monate definiert (z.B. angewendet in HONOR [107], IDEA [78], RETRO [49], STRASS [29]). Einige Studien verwenden auch die sog. anhaltend niedrige Krankheitsaktivität „sustained low-disease activity" mit einem DAS28 $<3,2$ als Einschlusskriterium (z. B. DRESS [118], DOSERA [121]).

Die Leitliniengruppe gibt zu bedenken, dass als Composite Score für die Definition der Remission in den Deeskalationsstudien die DAS28-Remission herangezogen wurde, die aber - wie bereits oben bei Empfehlung 2 dargelegt - möglicherweise als nicht ausreichend anzusehen ist. Es könnte daher nicht nur in Bezug auf die T2T-Strategie, sondern auch als Voraussetzung für die Deeskalation sinnvoll sein, eine anhaltende (also mindestens 6 Monate währende) SDAIRemission oder eine solche nach den Boole'schen Kriterien zu fordern, bevor eine Deeskalation vorgenommen wird. Daten dazu, ob die anhaltende SDAIRemission tatsächlich zu einer niedrigeren Rezidivrate bei Deeskalation als die DAS28-Remission führt, liegen jedoch nicht vor. 
Tab. 4 Risikofaktoren für ein erhöhtes Rezidivrisiko bei Deeskalation einer DMARD-Therapie

\begin{tabular}{|c|c|c|}
\hline Parameter & Referenz & Evidenzgrad \\
\hline \multicolumn{3}{|l|}{ Labor } \\
\hline Pos. Anti-CCP-Status & [83] (RETRO) & 2 \\
\hline Pos. RF IgM-Status & [29] (STRASS) & 2 \\
\hline „Multibiomarker disease activity state ${ }^{\prime a}$ & [49] (RETRO) & 2 \\
\hline \multicolumn{3}{|l|}{ Scores/klinische Parameter } \\
\hline Initial hoher DAS28-Score & {$[79]$} & 3 \\
\hline \multicolumn{3}{|l|}{ Therapie } \\
\hline "Spacing", Beendigung der Therapie & [29] (STRASS) & 2 \\
\hline \multicolumn{3}{|l|}{ Bildgebung } \\
\hline $\begin{array}{l}\text { Hoher initaler "global score" bei } \\
\text { PD+-Synovialitis }\end{array}$ & {$[79]$} & 3 \\
\hline Residuelle Synovialitis in der Remission & {$[58]$} & 3 \\
\hline \multicolumn{3}{|c|}{$\begin{array}{l}\text { PD Power Doppler, RF Rheumafaktor, Anti-CCP Antikörper gegen zyklische, citrullinierte Peptide } \\
\text { aMultibiomarker disease activity state = Status der Krankheitsaktivität gemessen an einem validierten } \\
\text { Score unter Bestimmung von } 12 \text { inflammatorischen Markern (EGF, VEGF-A, IL-6, SAA, CRP, MMP-1, } \\
\text { MMP-3, TNF-R1, YKL-40, Leptin und Resistin) im Serum des Patienten [83] }\end{array}$} \\
\hline
\end{tabular}

Tab. 5 Faktoren welche mit einer anhaltenden Remission oder LDA nach Tapering/Absetzen von DMARDs assoziiert sind

\begin{tabular}{lll}
\hline Parameter & Referenz & Evidenzgrad \\
\hline $\begin{array}{l}\text { Scores/klinische Parameter } \\
\text { LDA beim Beginn des Taperings (conventional DMARD) }\end{array}$ & {$[58]$} & 2
\end{tabular}

- Tab. 4 fasst die wichtigsten Parameter zusammen, die bisher als Faktoren für ein erhöhtes Flarerisiko identifiziert werden konnten und $\bullet$ Tab. 5 diejenigen Parameter, die mit einer anhaltenden Remission oder anhaltend niedrigen Krankheitsaktivität nach Dosisreduktion und/ oder Absetzen von DMARDs assoziiert sind.

Bisher liegen nur wenige Studien vor, welche Risikofaktoren für ein Flare bzw. Faktoren für eine anhaltende Remission unter Deeskalation untersucht haben (s. Forschungs-Agenda). Die Studien kamen teilweise zu widersprüchlichen Ergebnissen. Bei den bisher vorliegenden Studien handelt es sich zumeist um RCTs, in denen bestimmte Deeskalationsstrategien miteinander verglichen wurden und die Patienten in den jeweiligen Therapiearmen nach möglichen Risikofaktoren (z. B. Anti-CCP-Antikörper-Status) stratifiziert wurden oder um unkontrollierte Studien. Damit ist die Evidenzlage als Grad 2 bzw. 3 zu bewerten.

\section{Ablauf einer Deeskalation von DMARDs}

Zum Zeitpunkt der Literatursuche wurde in den bisher publizierten Deeskalationsstudien fast ausschließlich die Deeskalation der bDMARDs bei Patienten untersucht, die eine Kombinationstherapie aus csDMARDs und bDMARDs erhielten. Aufgrund dieser Datenlage ist folgendes festzustellen:

Die Deeskalation von bDMARDs könnte bei Patienten sinnvoll sein, die parallel mit einem csDMARD (i.d.R. MTX) behandelt werden (Empfehlungs$\operatorname{grad}$ D).

Generell kann aber eine Empfehlung, in welcher Reihenfolge die DMARDs reduziert werden sollen, aufgrund einer fehlenden Datenlage (also von Studien, die das Outcome einer bDMARD vs. csDMARD-Deeskalation vergleichen) nicht gegeben werden (s. ForschungsAgenda).

In der CAMEO-Studie wurde MTX in einem Studienarm im Rahmen der Deeskalation beendet. Diese Strategie war der unveränderten Fortführung der Therapie aus MTX/Etanercept nicht gleichwertig. Ein Vergleich zum Absetzen von
Etanercept unter Fortführung von MTX wurde allerdings nicht durchgeführt [61]. So könnte es ebenso sinnvoll sein bei Patienten mit einer bDMARD- und csDMARD-Kombination das csDMARD zuerst zu deeskalieren.

Eine Deeskalation sollte grundsätzlich in Form eines „Taperings“ (Dosisreduktion) oder "Spacings“ (Verlängerung der Applikationsintervalle) erfolgen. Ein sofortiges Absetzen der DMARD-Therapie wird nicht empfohlen (Empfehlungsgrad C). Die Deeskalation sollte unter engmaschiger rheumatologischer Verlaufskontrolle vorgenommen werden (Empfehlungsgrad D).

Bei fehlenden konkreten Empfehlungen erfolgte in den meisten hierzu vorliegenden Studien eine Halbierung der Dosis entweder durch Applikation der halben bDMARD-Dosis unter Beibehaltung des Applikationsintervalls oder durch eine Verdoppelung des Applikationsintervalls. Bei diesem Vorgehen besteht ein gering erhöhtes Risiko für ein Rezidiv im Vergleich zur unveränderten Fortsetzung der bDMARD-Therapie (z.B. PRESERVE: LDA: 82,6 vs. 79,1\% bei Fortführung vs. Dosisreduktion von ETA auf $25 \mathrm{mg} /$ Woche oder DOSERA: SR/LDA $52 \%$ vs. $44 \%$ bei Fortführung vs. Dosisreduktion von ETA auf $25 \mathrm{mg} /$ Woche) [6, 28, 29, 49, 98, 121]. Ein komplettes Absetzen der bDMARDs führte in den vorliegenden Studien zu hohen Rezidivraten von ca. 40 bis $80 \%$ nach 6-18 Monaten und ist daher nur in Ausnahmefällen zu erwägen [6, 26, 28, 39, 49, 55, 58, 67, 98, 107, 121].

Besteht eine ausschließliche csDMARD- oder eine ausschließliche bDMARD-Therapie, so kann bei anhaltender Remission ebenfalls eine Deeskalation dieser Therapie erwogen werden. Dies bedeutet im Falle der ausschließlichen Therapie mit einem csDMARD nicht das komplette Absetzen der Therapie, was nur sehr selten möglich sein wird, wohl aber die Ermittlung der individuell notwendigen Dosis zum Erhalt einer Remission.

\section{Abbruch der Deeskalation und Reeskalation}

Im Falle eines Flares unter Deeskalation kann durch Wiederaufnahme der ur- 
sprünglich verabreichten Dosis der Vortherapie in der Regel eine erneute $\mathrm{Re}$ mission erreicht werden (Empfehlungs$\operatorname{grad}$ A).

In Studien zur Deeskalation wurde diese bei Auftreten eines Flares beendet und die Therapie wieder intensiviert, d. h. wenn ein Wiederaufflammen der Erkrankung festgestellt wurde [55, 115].

Eine einheitliche Definition für den Flare existiert bisher nicht. Die OMERACT-Arbeitsgruppe hat ein "Core Set“ von Parametern definiert, die in der Definition eines Flares Berücksichtigung finden sollen ([14]; siehe Glossar, • Tab. 1). In der klinischen Praxis entspricht ein Flare einer Zunahme der Krankheitsaktivität, die den Arzt veranlasst, die Therapie wieder zu eskalieren (siehe Glossar, - Tab. 1). Darüber hinaus existiert eine DAS28-basierte Definition, die einen Flare als Anstieg des DAS28 zwischen zwei Untersuchungen um 1,2 Punkte (oder 0,6 bei aktuellem DAS28 gröBer 3,2) beschreibt, also die Umkehr der EULAR-Response-Definition [21]. Die Verwendung des DAS28-Score für die Definition einer Krankheitsaktivität ist aus den oben genannten Gründen möglicherweise nicht optimal. Die Leitliniengruppe empfiehlt daher die im Glossar verwendete Definition des Verlusts von Remission oder niedriger Erkrankungsaktivität durch Anstieg der Krankheitsaktivität, gemessen anhand von Composite Scores, über eine tägliche Variation der Beschwerden hinaus. Diese Empfehlung ist nicht evidenzbasiert (Empfehlungsgrad D).

\section{Diskussion}

Die hier beschriebene neue S2e-Leitlinie ist das Ergebnis eines Konsensprozesses deutscher Rheumatologen/-innen mit systematischer Bewertung der aktuellen Evidenz zur Behandlung der RA. Sie lehnt sich an die aktuell ebenfalls neu überarbeitete EULAR-Empfehlungen [97] an, setzt in manchen Punkten jedoch auch andere Akzente. Gegenüber früheren Leitlinien wurden einige methodische Neuerung eingeführt: so wurden die Interessenkonflikte aller Teilnehmer der Leitliniengruppe von Ombudspersonen, welche die DGRh bestimmt hat, bewertet und der Konsensprozess wurde jeweils von unabhängigen Moderatoren der Arbeitsgemeinschaft der medizinischwissenschaftlichen Fachgesellschaften (AWMF) geleitet. Beide Maßnahmen dienten dem Ziel, einen möglichst unabhängigen und objektiven Konsensprozess $\mathrm{zu}$ erreichen. Inhaltlich wurde erstmals der Stellenwert der JAK-Inhibitoren definiert und detaillierter als früher haben wir diskutiert, welche Daten existieren, um Therapieentscheidungen im Sinne einer individualisierten Medizin und für eine Deeskalation der DMARD-Therapien zu treffen. Auch inhaltlich spiegelt diese neue Leitlinie im Vergleich zu der vorherigen [65] neben einer gewissen Kontinuität auch viele Neuentwicklungen wider.

Die wichtigste Absicht der Autoren dieser Leitlinie ist es, dem Rheumatologen auf der Basis der vorhandenen Evidenz Entscheidungshilfen für das therapeutische Vorgehen auf dem aktuellsten Stand zur Verfügung zu stellen. Es ist zu hoffen, dass im nächsten Schritt die Inhalte der LL tatsächlich in die Versorgung der RA-Patienten implementiert werden. Gegenwärtig bestehen bei der Umsetzung des T2T-Prinzips hierzulande noch Defizite: Eine aktuelle Auswertung der Kerndokumentation der Deutschen Rheumazentren hat gezeigt [2], dass z. B. bei jedem zweiten erfassten RAPatienten bei Anwendung des T2T-Prinzips eine Therapiemodifizierung vorgenommen werden müsste, da das Therapieziel einer Remission oder LDA nicht erreicht ist. Auch die CAPEA-Studie [1], die Auswertung des zweijährigen Krankheitsverlaufes einer Früharthritiskohorte in Deutschland, belegt Defizite: So änderte sich der Anteil der Patienten mit moderater oder hoher Krankheitsaktivität in dieser Studie zwischen Monat 6 und 24 nicht, er lag nach 2 Jahren noch immer bei $37 \%$. Dennoch erhielten im Gesamtverlauf nur $10 \%$ eine csDMARDKombination und $12 \%$ ein bDMARD, $47 \%$ der Patienten standen noch unter GC.

Auch wenn die Prinzipien des evidenzbasierten Vorgehens so weit wie möglich bei der Erstellung der LL angewendet wurden, hat sich gezeigt, dass nach wie vor längst nicht alle relevan- ten Fragen wirklich evidenzbasiert zu beantworten sind, da entsprechende Studien fehlen. Für die Zukunft bleibt somit viel weitere Arbeit zu leisten. Die Leitliniengruppe hat versucht, dem mit einer Forschungs-Agenda Rechnung zu tragen.

\section{Forschungs-Agenda}

\section{Therapiealgorithmus}

- Sollte eine DMARD-Therapie bereits im Frühstadium einer undifferenzierten Arthritis, bei Risikofaktoren für die Entwicklung einer RA, auch ohne dass die ACR/EULAR Klassifikationskriterien erfüllt sind, begonnen werden und wenn ja wie?

- Ist der Beginn einer DMARDTherapie auch bei sehr geringer Aktivität der RA sinnvoll?

- Gibt es Situationen/Konstellationen, in denen eine Starttherapie mit hierfür zugelassenen bDMARDs zu empfehlen ist?

- Wie ist die Wirksamkeit und Sicherheit anderer csDMARDs, wie Leflunomid und Sulfasalzin, im Vergleich zu MTX in der initialen Therapie und als Kombinationspartner mit bDMARDs und tsDMARDs?

- Wie ist die Wirksamkeit und Sicherheit oraler Gaben von Glukokortikoiden sowohl in der Therapie initial (z. B. Prednisolonäquivalent 10-30 mg/Tag) als auch im Verlauf z. B. bei der Therapie eines Flares

- Ist eine Therapie mit intraartikulären GC zusätzlich zur Therapie mit DMARDs effektiv und wie viele intraartikuläre Injektionen pro Gelenk sind in welchem Zeitraum sinnvoll und unbedenklich?

- Wie valide sind die Kriterien zur Auswahl von Patienten für b- oder tsDMARD-Therapie versus DMARD-Kombination nach inadäquatem Ansprechen auf das erste DMARD?

- Ist das Langzeit-Outcome für den Einsatz von bDMARDs oder tsDMARDs nach dem ersten csDMARD im Vergleich zur dem nach einer zweiten csDMARDStrategie unterschiedlich? Haben 
Risikofaktoren (ACPA-Positivität, Erosivität usw.) einen Einfluss?

- Wie ist die Wirksamkeit und Sicherheit von b- oder tsDMARDs, wenn als erstes bDMARD kein TNFInhibitor gegeben wurde?

- Wie ist die Präferenz und Adhärenz von oralen tsDMARDs im Vergleich $\mathrm{zu}$ bDMARDs und in Kombination mit csDMARDs?

- Was sind die Gründe und Mechanismen für den sekundären Wirkverlust von DMARDs und wie kann dieser vermieden werden?

- Was bewirken DMARDs bzw. DMARD-Kombinationen im Hinblick auf verschiedene Komorbiditäten, z. B. das kardiovaskuläre Risiko, pulmonale Manifestationen, Diabetes u. a.?

\section{Treat-to-Target (T2T)}

- Ist T2T bei allen RA Patienten, z. B. auch bei hochbetagten und/oder erheblich komorbiden Patienten, sinnvoll?

- Welche Möglichkeiten der engmaschigen Verlaufsbeurteilung im initialen Therapiestadium der RA gibt es und welche Rolle kann a) Telemedizin und b) Delegation an rheumatologische Fachassistenten dabei spielen?

- Welche Rolle spielen MRT und Sonographie in der Verlaufsbeurteilung des T2T?

- Wie kann eine Verbesserung der Adhärenz zu T2T in der rheumatologischen Routineversorgung bewirkt werden?

\section{Biomarker und Individualisierte Medizin}

- Welche Rolle spielen genetische oder immunologische Marker für die Prognose der RA oder die Therapieentscheidungen?

- Gibt es Prädiktoren für das Ansprechen oder das Nicht-Ansprechen auf ein bestimmtes bDMARD oder tsDMARD?

- Welche Prädiktoren gibt es für das Erreichen des Therapieziels früh im Verhandlungsverlauf?
- Welche Rolle kann die Messung von „anti-drug antibodies“ (ADA) und/ oder von Medikamentenkonzentrationen im Blut in der klinischen Praxis für die Festlegung der Dosis oder des Dosierungsintervalls von DMARDs spielen?

- Welches sind die Prädiktoren für das Auftreten von unerwünschten Ereignissen?

\section{Deeskalation}

- Wie ist die Kosten-Nutzen-Analyse einer Deeskalationstherapie bei RA?

- Welche Prädiktoren gibt es für eine erfolgreiche Deeskalation und für einen Flare?

- Was ist die richtige Deeskalationsstrategie bezüglich der Reihenfolge der zu deeskalierenden DMARDs (bDMARD, tsDMARD oder csDMARD)?

- Was ist die richtige Deeskalationsstrategie? Dosisreduktion oder Spacing, rasche oder langsame Deeskalation, maximal Halbierung der Dosis oder weitere Dosisreduktion?

\section{Korrespondenzadresse}

\section{Prof. Dr. med. C. Fiehn}

Praxis für Rheumatologie, Tätigkeitsschwerpunkt Klinische Immunologie und Belegarzteinheit der ViDia-Kliniken Karlsruhe am Medical Center Baden-Baden Beethovenstr. 2, 76530 Baden-Baden, Deutschland

c.fiehn@rheuma-badenbaden.de

Danksagung. Wir danken Frau Ekatharina Suponitskaya für ihre wertvolle Unterstützung bei der Selektion der Abstracts im Rahmen der systematischen Literaturrecherche und den von der DGRh benannten Ombudspersonen E. Genth, Aachen, W. Gross, Lübeck und H.-H. Peter, Freiburg für die Prüfung der Interessenkonflikte.

\section{Einhaltung ethischer Richtlinien}

Interessenkonflikt. Angaben zum Interessenskonflikt aller Leitlinienautoren sind im Leitlinienreport unter http://www.awmf.org/leitlinien/detail/ anmeldung/1/ll/060-004.html einsehbar.

Dieser Beitrag beinhaltet keine von den Autoren durchgeführten Studien an Menschen oder Tieren.

\section{Literatur}

1. Albrecht K, Callhoff J, Edelmann E et al (2016) Clinical remission in rheumatoid arthritis. Data from the early arthritis cohort study CAPEA. ZRheumatol 75:90-96

2. Albrecht K, Huscher D, Eidner Tet al (2017) Medical treatment of rheumatoid arthritis in 2014: current data from the German collaborative arthritis centers. ZRheumatol 76:50-57

3. Aletaha D, Alasti F, Smolen JS (2016) Optimisation of a treat-to-target approach in rheumatoid arthritis: strategies for the 3-month time point. Ann Rheum Dis 75:1479-1485

4. Aletaha D, Funovits J, Keystone EC et al (2007) Disease activity early in the course of treatment predicts response to therapy after one year in rheumatoid arthritis patients. Arthritis Rheum 56:3226-3235

5. Aletaha D,NeogiT,Silman AJetal (2010) 2010 rheumatoid arthritis classification criteria: an American College of Rheumatology/European League Against Rheumatism collaborative initiative. Ann Rheum Dis 69:1580-1588

6. Alivernini S, Peluso G, Fedele AL et al (2016) Tapering and discontinuation of TNF-alpha blockers without disease relapse using ultrasonography as a tool to identify patients with rheumatoid arthritis in clinical and histological remission. Arthritis Res Ther 18:39

7. Andersson ML, Bergman S, Soderlin MK (2012) The effect of stopping smoking on disease activity in rheumatoid arthritis (RA). Data from BARFOT, a multicenter study of early RA. Open Rheumatol J 6:303-309

8. Barnabe C, Martin BJ, Ghali WA (2011) Systematic review and meta-analysis: anti-tumor necrosis factor alpha therapy and cardiovascular events in rheumatoid arthritis. Arthritis Care Res (Hoboken) 63:522-529

9. Braun J, Kastner P, Flaxenberg $P$ et al (2008) Comparison of the clinical efficacy and safety of subcutaneous versus oral administration of methotrexate in patients with active rheumatoid arthritis: results of a six-month, multicenter, randomized, double-blind, controlled, phase IV trial. Arthritis Rheum 58:73-81

10. Burmester GR, Blanco R, Charles-Schoeman $C$ et al (2013) Tofacitinib (CP-690,550) in combination with methotrexate in patients with active rheumatoid arthritis with an inadequate response to tumour necrosis factor inhibitors: a randomised phase 3 trial. Lancet 381:451-460

11. Burmester GR, Kivitz AJ, Kupper $\mathrm{H}$ et al (2015) Efficacy and safety of ascending methotrexate dose in combination with adalimumab: the randomised CONCERTO trial. Ann Rheum Dis 74:1037-1044

12. Burmester GR, Lin Y, Patel R et al (2017) Efficacy and safety of sarilumab monotherapy versus adalimumab monotherapy for the treatment of patients with active rheumatoid arthritis (MONARCH): a randomised, double-blind, parallelgroup phase III trial. Ann Rheum Dis 76:840-847

13. Burmester GR, Rigby WF, Van Vollenhoven RF et al (2016) Tocilizumab in early progressive rheumatoid arthritis: FUNCTION, a randomised controlled trial. Ann Rheum Dis 75:1081-1091

14. Bykerk VP, Lie E, Bartlett SJ et al (2014) Establishing a core domain set to measure rheumatoid arthritis flares:report of the OMERACT 11RAflareworkshop. JRheumatol 41:799-809

15. Chatzidionysiou K, Emamikia S, Nam J et al (2017) Efficacy of glucocorticoids, conventional and tar- 
geted synthetic disease-modifying antirheumatic drugs: a systematic literature review informing the 2016 update of the EULAR recommendations for the management of rheumatoid arthritis. Ann Rheum Dis 76:1102-1107

16. Combe B, Landewe R, Daien Cl et al (2017) 2016 update of the EULAR recommendations for the management of early arthritis. Ann Rheum Dis 76:948-959

17. Curtis JR, Luijtens K, Kavanaugh A (2012) Predicting future response to certolizumab pegol in rheumatoid arthritis patients: features at 12 weeks associated with low disease activity at 1 year. Arthritis Care Res (hoboken) 64:658-667

18. Dale J, Purves D, Mcconnachie A et al (2014) Tightening up? Impact of musculoskeletal ultrasound disease activity assessment on early rheumatoid arthritis patients treated using a treat to target strategy. Arthritis Care Res (hoboken) 66:19-26

19. De Jong PH, Hazes JM, Han HKet al (2014) Randomised comparison of initial triple DMARD therapy with methotrexate monotherapy in combination with low-dose glucocorticoid bridging therapy; 1 -year data of the tREACH trial. Ann Rheum Dis 73:1331-1339

20. Den Uyl D, Ter Wee M, Boers M et al (2014) A non-inferiority trial of an attenuated combination strategy ('COBRA-light') compared to the original COBRA strategy: clinical results after 26 weeks. Ann Rheum Dis 73:1071-1078

21. Dougados M, Huizinga TW, Choy EH et al (2015) Evaluation of the disease activity score in twentyeight joints-based flare definitions in rheumatoid arthritis: data from a three-year clinical trial. Arthritis Care Res (hoboken) 67:1762-1766

22. Dougados M, Kissel K, Conaghan PG et al (2014) Clinical, radiographic and immunogenic effects after 1 year of tocilizumab-based treatment strategies in rheumatoid arthritis: the ACT-RAY study. Ann Rheum Dis 73:803-809

23. Dougados M, Van Der Heijde D, Chen YCetal (2017) Baricitinib in patients with inadequate response or intolerance to conventional synthetic DMARDs: results from the RA-BUILD study. Ann Rheum Dis 76:88-95

24. Duru N, Van Der Goes MC, Jacobs JW et al (2013) EULAR evidence-based and consensusbased recommendations on the management of medium to high-dose glucocorticoid therapy in rheumatic diseases. Ann Rheum Dis 72:1905-1913

25. Emery P, Bingham CO 3rd, Burmester GRetal (2017) Certolizumab pegol in combination with doseoptimised methotrexate in DMARD-naive patients with early, active rheumatoid arthritis with poor prognostic factors: 1-year results from C-EARLY, a randomised, double-blind, placebo-controlled phase Ill study. Ann Rheum Dis 76:96-104

26. Emery P, Burmester GR, Bykerk VP et al (2015) Evaluating drug-free remission with abatacept in early rheumatoid arthritis: results from the phase $3 \mathrm{~b}$, multicentre, randomised, active-controlled AVERT study of 24 months, with a 12-month, double-blind treatment period. Ann Rheum Dis 74:19-26

27. Emery P, Gottenberg JE, Rubbert-Roth A etal (2015) Rituximab versus an alternative TNF inhibitor in patients with rheumatoid arthritis who failed to respond to a single previous TNF inhibitor: SWITCH-RA, a global, observational, comparative effectiveness study. Ann Rheum Dis 74:979-984

28. Emery $P$, Hammoudeh M, Fitzgerald $O$ et al (2014) Sustained remission with etanercept tapering in early rheumatoid arthritis. N Engl J Med 371:1781-1792
29. Fautrel B, Pham T, Alfaiate T et al (2016) Step-down strategy of spacing TNF-blocker injections for established rheumatoid arthritis in remission:results of the multicentrenon-inferiority randomised open-label controlled trial (STRASS: Spacing of TNF-blocker injections in Rheumatoid ArthritiS Study). Ann Rheum Dis 75:59-67

30. Felson DT, Smolen JS, Wells G et al (2011) American College of Rheumatology/European League against Rheumatism provisional definition of remission in rheumatoid arthritis for clinical trials. Ann Rheum Dis 70:404-413

31. Fiehn C, Belke-Voss E, Krause D et al (2013) Improved radiological outcome of rheumatoid arthritis: the importance of early treatment with methotrexate in the era of biological drugs. Clin Rheumatol 32:1735-1742

32. Fiehn C, Herzer P, Holle J et al (2016) Klug entscheiden in der Rheumatologie. Dtsch Arzteb 113:A-1154 (B-1939/C-1953)

33. Finckh A, Choi HK, Wolfe F (2006) Progression of radiographic joint damage in different eras: trends towards milder disease in rheumatoid arthritis are attributable to improved treatment. Ann Rheum Dis 65:1192-1197

34. Fleischmann R, Kremer J, Cush J et al (2012) Placebo-controlled trial of tofacitinib monotherapy in rheumatoid arthritis. NEngl J Med 367:495-507

35. Fleischmann R, Mysler E, Hall S et al (2017) Efficacy and safety of tofacitinib monotherapy, tofacitinib with methotrexate, and adalimumab with methotrexate in patients with rheumatoid arthritis (ORAL Strategy): a phase 3b/4, doubleblind, head-to-head, randomised controlled trial. Lancet 390:457-468

36. Fleischmann R, Schiff M, Van Der Heijde D et al (2017) Baricitinib, Methotrexate, or combination in patients with rheumatoid arthritis and no or limited prior disease-modifying antirheumatic drug treatment. Arthritis Rheumatol 69:506-517

37. Gabay C, Emery P, Van Vollenhoven R et al (2013) Tocilizumab monotherapy versus adalimumab monotherapy for treatment of rheumatoid arthritis (ADACTA): a randomised, double-blind, controlled phase 4 trial. Lancet 381:1541-1550

38. Genovese MC, Kremer J, Zamani O et al (2016) Baricitinib in patients with refractory rheumatoid arthritis. NEngl J Med 374:1243-1252

39. Ghiti Moghadam M, Vonkeman HE, Ten Klooster PM et al (2016) Stopping tumor necrosis factor inhibitor treatment in patients with established rheumatoid arthritis in remission or with stable low disease activity: a pragmatic multicenter, open-label randomized controlled trial. Arthritis Rheumatol 68:1810-1817

40. Glintborg B, Sorensen IJ, Loft AG et al (2017) A nationwide non-medical switch from originator infliximab to biosimilar CT-P13 in 802 patients with inflammatory arthritis: 1-year clinical outcomes from the DANBIO registry. Ann Rheum Dis 76:1426-1431

41. Goekoop-Ruiterman YP, De Vries-Bouwstra JK, Allaart CF et al (2007) Comparison of treatment strategies in early rheumatoid arthritis: a randomized trial. Ann Intern Med 146:406-415

42. Golicki D, Newada M, Lis J et al (2012) Leflunomide in monotherapy of rheumatoid arthritis: metaanalysis of randomized trials. Pol Arch Med Wewn 122:22-32

43. Gottenberg JE, Brocq O, Perdriger A et al (2016) Non-TNF-targeted biologic vs a second anti-TNF drug to treat rheumatoid arthritis in patients with insufficient response to a first anti-TNF drug: a randomized clinical trial. JAMA 316:1172-1180
44. Gottenberg JE, Courvoisier DS, Hernandez MV et al (2016) Brief report: association of rheumatoid factor and anti-citrullinated protein antibody positivity with better effectiveness of Abatacept: results from the Pan-European Registry Analysis. Arthritis Rheumatol 68:1346-1352

45. Gremese E, Gigante MR, Tolusso B et al (2015) Weight loss in obese rheumatoid arthritis (RA) patients improves disease activity without modifying RA treatment. Arthritis Rheumatol 67(Suppl 10). http://acrabstracts.org/abstract/weightloss-in-obese-rheumatoid-arthritis-ra-patientsimproves-disease-activity-without-modifyingra-treatment/.Zugegriffen:24.05.2018

46. Haavardsholm EA, Aga AB, Olsen IC et al (2016) Ultrasound in management of rheumatoid arthritis: ARCTIC randomised controlled strategy trial.BMJ 354:i4205

47. Harrold LR, Reed GW, Kremer JM et al (2015) The comparative effectiveness of abatacept versus anti-tumour necrosis factor switching for rheumatoid arthritis patients previously treated with an anti-tumour necrosis factor. Ann Rheum Dis 74:430-436

48. Harrold LR, Reed GW, Solomon DH et al (2016) Comparative effectiveness of abatacept versus tocilizumab in rheumatoid arthritis patients with prior TNFi exposure in the US Corrona registry. Arthritis Res Ther 18:280

49. Haschka J, Englbrecht $M$, Hueber AJ et al (2016) Relapse rates in patients with rheumatoid arthritis in stable remission tapering or stopping antirheumatic therapy: interim results from the prospective randomised controlled RETRO study. Ann Rheum Dis 75:45-5

50. Hazlewood GS, Barnabe C, Tomlinson G et al (2016) Methotrexate monotherapy and methotrexate combination therapy with traditional and biologic disease modifying antirheumatic drugs for rheumatoid arthritis: abridged Cochrane systematic review and network meta-analysis. BMJ 353:i1777

51. Hazlewood GS, Thorne JC, Pope JE et al (2016) The comparative effectiveness of oral versus subcutaneous methotrexate for the treatment of early rheumatoid arthritis. Ann Rheum Dis 75:1003-1008

52. Heimans L, Wevers-De Boer KV, Visser Ket al (2014) A two-step treatment strategy trial in patients with early arthritis aimed at achieving remission: the IMPROVED study. Ann Rheum Dis 73:1356-1361

53. Hetland ML, Horslev-Petersen K (2012) The CIMESTRA study: intra-articular glucocorticosteroids and synthetic DMARDs in a treat-to-target strategy in early rheumatoid arhtritis. Clin Exp Rheumatol 30:\$44-49

54. Hoes JN, Jacobs JW, Boers M et al (2007) EULAR evidence-based recommendations on the management of systemic glucocorticoid therapyin rheumatic diseases. Ann Rheum Dis 66:1560-1567

55. Huizinga TW, Conaghan PG, Martin-Mola E et al (2015) Clinical and radiographic outcomes at 2 years and the effect of tocilizumab discontinuation following sustained remission in the second and third year of the ACT-RAY study. Ann Rheum Dis 74:35-43

56. Huscher D, Sengler C, Gromnica-Ihle E et al (2013) Clinical presentation, burden of disease and treatment in young-onset and late-onset rheumatoid arthritis: a matched-pairs analysis taking age and disease duration into account. Clin Exp Rheumatol 31:256-262

57. Isaacs JD, Cohen SB, Emery P et al (2013) Effect of baseline rheumatoid factor and anticitrullinated peptide antibody serotype on rituximab clinical 
response: a meta-analysis. Ann Rheum Dis 72:329-336

58. Iwamoto T, Ikeda K, Hosokawa J et al (2014) Prediction of relapse after discontinuation of biologic agents by ultrasonographic assessment in patients with rheumatoid arthritis in clinical remission: high predictivevalues of total gray-scale and power Doppler scores that represent residual synovial inflammation before discontinuation. Arthritis Care Res (Hoboken) 66:1576-1581

59. Kaneko Y, Atsumi T, Tanaka Y et al (2016) Comparison of adding tocilizumab to methotrexate with switching to tocilizumab in patients with rheumatoid arthritis with inadequate response to methotrexate: 52-week results from a prospective, randomised, controlled study (SURPRISE study). Ann Rheum Dis 75:1917-1923

60. Kay J, Schoels MM, DornerT et al (2017) Consensus based recommendations for the use of biosimilars to treat rheumatological diseases. Ann Rheum Dis. https://doi.org/10.1136/annrheumdis-2017211937

61. Keystone EC, Pope JE, Thorne JC et al (2016) Twoyear radiographic and clinical outcomes from the Canadian Methotrexate and Etanercept Outcome study in patients with rheumatoid arthritis. Rheumatology (Oxford) 55:327-334

62. Kiely P, Walsh D, Williams R et al (2011) Outcome in rheumatoid arthritis patients with continued conventional therapy for moderate disease activity-the early RA network (ERAN). Rheumatology (Oxford) 50:926-931

63. KlarenbeekNB, Guler-Yuksel M, Van Der KooijSMet al (2011) The impact of four dynamic, goal-steered treatment strategies on the 5-year outcomes of rheumatoid arthritis patients in the BeSt study. Ann Rheum Dis 70:1039-1046

64. Kremer J, Li ZG, Hall S et al (2013) Tofacitinib in combination with nonbiologic disease-modifying antirheumatic drugs in patients with active rheumatoid arthritis: a randomized trial. Ann Intern Med 159:253-261

65. Kruger K, Wollenhaupt J, Albrecht K et al (2012) German 2012 guidelines for the sequential medical treatment of rheumatoid arthritis. Adapted EULAR recommendations and updated treatment algorithm. ZRheumatol 71:592-603

66. Lee EB, Fleischmann R, Hall Set al (2014) Tofacitinib versus methotrexate in rheumatoid arthritis. NEngl J Med 370:2377-2386

67. Li R, Zhao JX, Su Y et al (2016) High remission and low relapse with prolonged intensive DMARD therapy in rheumatoid arthritis (PRINT): a multicenter randomized clinical trial. Medicine (Baltimore) 95:e3968

68. Listing J, Gerhold K, Zink A (2013) The risk of infections associated with rheumatoid arthritis, with its comorbidity and treatment. Rheumatology (Oxford) 52:53-61

69. Listing J, Kekow J, Manger B et al (2015) Mortality in rheumatoid arthritis: the impact of disease activity, treatment with glucocorticoids, TNFalpha inhibitors and rituximab. Ann Rheum Dis 74:415-421

70. Lofland JH, Johnson PT, Ingham MP et al (2017) Shared decision-making for biologic treatment of autoimmune disease: influence on adherence, persistence, satisfaction, and health care costs. Patient Prefer Adherence 11:947-958

71. Lopez-Olivo MA, Siddhanamatha HR, Shea B et al (2014) Methotrexate for treating rheumatoid arthritis. Cochrane Database Syst Rev. https://doi. org/10.1002/14651858.cd000957.pub2
72. Lupoli R, Pizzicato P, Scalera A et al (2016) Impact of body weight on the achievement of minimal disease activity in patients with rheumatic diseases: a systematic review and meta-analysis. Arthritis Res Ther 18:297

73. Manders SH, Kievit W, Jansen TL et al (2016) Effectiveness of tumor necrosis factor inhibitors in combination with various csDMARD in the treatment of rheumatoid arthritis: data from the DREAM registry. J Rheumatol 43:1787-1794

74. Mandl P, Balint PV, Brault Y et al (2013) Clinical and ultrasound-based composite disease activity indices in rheumatoid arthritis: results from a multicenter, randomized study. Arthritis Care Res (hoboken) 65:879-887

75. Maneiro JR, Salgado E, Gomez-Reino JJ (2013) Immunogenicity ofmonoclonal antibodiesagainst tumor necrosis factor used in chronic immunemediated Inflammatory conditions: systematic review and meta-analysis. Jama Intern Med 173:1416-1428

76. Mouterde G, Baillet A, Gaujoux-Viala C et al (2011) Optimizing methotrexate therapy in rheumatoid arthritis: a systematic literature review. Joint Bone Spine 78:587-592

77. Nam JL, Takase-Minegishi K, Ramiro $S$ et al (2017) Efficacy of biological disease-modifying antirheumatic drugs: a systematic literature review informing the 2016 update of the EULAR recommendations for the management of rheumatoid arthritis. Ann Rheum Dis 76:1113-1136

78. Nam JL, Villeneuve E, Hensor EM et al (2014) Remission induction comparing infliximab and high-dose intravenous steroid, followed by treatto-target: a double-blind, randomised, controlled trial in new-onset, treatment-naive, rheumatoid arthritis (the IDEA study). Ann Rheum Dis 73:75-85

79. NaredoE, Valor L, De La Torre letal (2015) Predictive value of Doppler ultrasound-detected synovitis in relation to failed tapering of biologic therapy in patients with rheumatoid arthritis. Rheumatology (Oxford) 54:1408-1414

80. Ornbjerg LM, Ostergaard M, Boyesen $P$ et al (2014) Which factors influence radiographic progression during treatment with tumor necrosis factor inhibitors in clinical practice? Results from 930 patients with rheumatoid arthritis in the nationwide Danish DANBIO registry. J Rheumatol 41:2352-2360

81. Pasma A, Hazes JM, Busschbach JJ et al (2017) Psychosocial predictors of DMARD adherence in the first three months of treatment for early arthritis. Patient Educ Couns 100:126-132

82. Ramiro S, Sepriano A, Chatzidionysiou K et al (2017) Safety of synthetic and biological DMARDs: a systematic literature review informing the 2016 update of the EULAR recommendations for management of rheumatoid arthritis. Ann Rheum Dis 76:1101-1136

83. Rech J, Hueber AJ, Finzel S et al (2016) Prediction of disease relapses by multibiomarker disease activity and autoantibody status in patients with rheumatoid arthritis on tapering DMARD treatment. Ann Rheum Dis 75:1637-1644

84. Rezaei H, Saevarsdottir S, Forslind K et al (2012) In early rheumatoid arthritis, patients with a good initial response to methotrexate have excellent 2-year clinical outcomes, but radiological progression is not fully prevented: data from the methotrexate responders population in the SWEFOT trial. Ann Rheum Dis 71:186-191

85. Saevarsdottir S, Rezaei H, Geborek P et al (2015) Current smoking status is a strong predictor of radiographic progression in early rheumatoid arthritis: results from the SWEFOT trial. Ann Rheum Dis 74:1509-1514

86. Saevarsdottir $S$, Wedren $S$, Seddighzadeh M et al (2011) Patients with early rheumatoid arthritis who smoke are less likely to respond to treatment with methotrexate and tumor necrosis factor inhibitors: observations from the Epidemiological Investigation of Rheumatoid Arthritis and the Swedish Rheumatology Register cohorts. Arthritis Rheum 63:26-36

87. Schiff MH, Jaffe JS, Freundlich B (2014) Head-tohead, randomised, crossover study of oral versus subcutaneous methotrexate in patients with rheumatoid arthritis: drug-exposure limitations of oral methotrexate at doses $\geq 15 \mathrm{mg}$ may be overcome with subcutaneous administration. Ann Rheum Dis 73:1549-1551

88. Sharma TS, Wasko MC, Tang X et al (2016) Hydroxychloroquine use is associated with decreased incident cardiovascular events in rheumatoid arthritis patients. J Am Heart Assoc. https://doi.org/10.1161/jaha.115.002867

89. Shea B, Swinden MV, Tanjong Ghogomu E et al (2013) Folic acid and folinic acid for reducing side effects in patients receiving methotrexate for rheumatoid arthritis. Cochrane Database Syst Rev. https://doi.org/10.1002/14651858. cd000951.pub2

90. Singh JA, Saag KG, Bridges SL Jr. et al (2016) 2015 American College of Rheumatology guideline for the treatment of rheumatoid arthritis. Arthritis Rheumatol 68:1-26

91. Smolen JS, Aletaha D (2015) Rheumatoid arthritis therapy reappraisal: strategies, opportunities and challenges. Nat Rev Rheumatol 11:276-289

92. Smolen JS, Breedveld FC, Burmester GR et al (2016) Treating rheumatoid arthritis to target: 2014 update of the recommendations of an international task force. Ann Rheum Dis 75:3-15

93. Smolen JS, Burmester GR, Combe B et al (2016) Head-to-head comparison of certolizumab pegol versus adalimumab in rheumatoid arthritis: 2-year efficacy and safety results from the randomised EXXELERATE study. Lancet 388:2763-2774

94. Smolen JS, Han C, Van Der Heijde DM et al (2009) Radiographic changes in rheumatoid arthritis patients attaining different disease activity states with methotrexate monotherapy and infliximab plus methotrexate: the impacts of remission and tumour necrosis factor blockade. Ann Rheum Dis 68:823-827

95. Smolen JS, Kalden JR, Scott DL et al (1999) Efficacy and safety of leflunomide compared with placebo and sulphasalazine in active rheumatoid arthritis: a double-blind, randomised, multicentre trial. European Leflunomide Study Group. Lancet 353:259-266

96. Smolen JS, Kay J, Doyle MKet al (2009) Golimumab in patients with active rheumatoid arthritis after treatment with tumour necrosis factor alpha inhibitors (GO-AFTER study): a multicentre, randomised, double-blind, placebo-controlled, phase III trial. Lancet 374:210-221

97. Smolen JS, Landewe R, Bijlsma J et al (2017) EULAR recommendations for the management of rheumatoid arthritis with synthetic and biological disease-modifying antirheumatic drugs: 2016 update. Ann Rheum Dis 76:960-977

98. Smolen JS, Nash P, DurezPetal (2013) Maintenance, reduction, or withdrawal of etanercept after treatment with etanercept and methotrexate in patients with moderate rheumatoid arthritis (PRESERVE): a randomised controlled trial. Lancet 381:918-929 
99. Smolen JS, Van Der Heijde DM, Clair StEW et al (2006) Predictors of joint damage in patients with early rheumatoid arthritis treated with highdose methotrexate with or without concomitant infliximab: results from the ASPIRE trial. Arthritis Rheum 54:702-710

100. Smolen JS, Wollenhaupt J, Gomez-Reino JJ et al (2015) Attainment and characteristics of clinical remission according to the new ACR-EULAR criteria in abatacept-treated patients with early rheumatoid arthritis: new analyses from the Abatacept study to gauge remission and joint damage progression in methotrexate (MTX)-naive patients with early erosive rheumatoid arthritis (AGREE). Arthritis Res Ther 17:157

101. Sokolove J, Schiff M, Fleischmann R et al (2016) Impact of baselineanti-cyclic citrullinated peptide2 antibody concentration on efficacy outcomes following treatment with subcutaneous abatacept or adalimumab: 2-year results from the AMPLE trial. Ann Rheum Dis 75:709-714

102. Sparks JA, Halperin F, Karlson JC et al (2015) Impact of bariatric surgery on patients with rheumatoid arthritis. Arthritis Care Res (hoboken) 67:1619-1626

103. Stoffer MA, Schoels MM, Smolen JS et al (2016) Evidence for treating rheumatoid arthritis to target: results of a systematic literature search update. Ann Rheum Dis 75:16-22

104. Stoffer MA, Smolen JS, Woolf A et al (2014) Development of patient-centred standards of care for rheumatoid arthritis in Europe: the eumusc.net project. Ann Rheum Dis 73:902-905

105. Strangfeld A, Eveslage M, Schneider M et al (2011) Treatment benefit or survival of the fittest: what drives the time-dependent decrease in serious infection rates under TNF inhibition and what does this imply for the individual patient? Ann Rheum Dis 70:1914-1920

106. Strehl C, Bijlsma JW, De Wit M et al (2016) Defining conditions where long-term glucocorticoid treatment has an acceptably low level of harm to facilitate implementation of existing recommendations: viewpoints from an EULAR task force. Ann Rheum Dis 75:952-957

107. Tanaka Y, Hirata S, Kubo S et al (2015) Discontinuation of adalimumab after achieving remission in patients with established rheumatoid arthritis: 1-year outcome of the HONOR study. Ann Rheum Dis 74:389-395

108. Taylor PC, Keystone EC, Van Der Heijde D et al (2017) Baricitinib versus placebo or Adalimumab in rheumatoid arthritis. N Engl J Med 376:652-662

109. Ter Wee MM, Lems WF, Usan $\mathrm{H}$ et al (2012) The effect of biological agents on work participation in rheumatoid arthritis patients: a systematic review. Ann Rheum Dis 71:161-171

110. Van Aken J, Heimans $L$, Gillet-Van Dongen $H$ et al (2014) Five-year outcomes of probable rheumatoid arthritis treated with methotrexate or placebo during the first year (the PROMPT study). Ann Rheum Dis 73:396-400

111. Van Der Heijde D, Aletaha D, Carmona $L$ et al (2015) 2014 Update of the EULAR standardised operating procedures for EULAR-endorsed recommendations. Ann Rheum Dis 74:8-13

112. Van Der Heijde D, Keystone EC, Curtis JR et al (2012) Timing and magnitude of initial change in disease activity score 28 predicts the likelihood of achieving low disease activity at 1 year in rheumatoid arthritis patients treated with certolizumab pegol: a post-hoc analysis of the RAPID 1 trial. J Rheumatol 39:1326-1333
113. Van Der Heijde D, Tanaka Y, Fleischmann R et al (2013) Tofacitinib (CP-690,550) in patients with rheumatoid arthritis receiving methotrexate: twelve-month data from a twenty-four-month phase III randomized radiographic study. Arthritis Rheum 65:559-570

114. Van Der Heijde DM, Van Riel PL, Van Leeuwen MA et al (1992) Prognostic factors for radiographic damage and physical disability in early rheumatoid arthritis. A prospective follow-up study of 147 patients. Br J Rheumatol 31:519-525

115. Van Der Maas A, Kievit W, Van Den Bemt BJ et al (2012) Down-titration and discontinuation of infliximab in rheumatoid arthritis patients with stable low disease activity and stable treatment: an observational cohort study. Ann Rheum Dis 71:1849-1854

116. Van Dongen H, Van Aken J, Lard LR et al (2007) Efficacy of methotrexate treatment in patients with probable rheumatoid arthritis: a doubleblind, randomized, placebo-controlled trial. Arthritis Rheum 56:1424-1432

117. Van Gestel AM, PrevooML, Van'THofMAetal (1996) Development and validation of the European League Against Rheumatism response criteria for rheumatoid arthritis. Comparison with the preliminary American College of Rheumatology and the World Health Organization/International League Against Rheumatism Criteria. Arthritis Rheum 39:34-40

118. Van Herwaarden N, Van Der Maas A, Minten MJ et al (2015) Disease activity guided dose reduction and withdrawal of adalimumab or etanercept compared with usual care in rheumatoid arthritis: open label, randomised controlled, non-inferiority trial. BMJ 350:h1389

119. Van Leeuwen MA, Van Rijswijk MH, Sluiter WJ et al (1997) Individual relationship between progression of radiological damage and the acute phase response in early rheumatoid arthritis. Towards development of a decision support system. JRheumatol 24:20-27

120. Van Vollenhoven RF, Fleischmann $R$, Cohen $S$ et al (2012) Tofacitinib or adalimumab versus placebo in rheumatoid arthritis. NEngl J Med 367:508-519

121. Van Vollenhoven RF, Ostergaard M, LeirisaloRepo M et al (2016) Full dose, reduced dose or discontinuation of etanercept in rheumatoid arthritis. Ann Rheum Dis 75:52-58

122. Verschueren P, De Cock D, Corluy L et al (2017) Effectiveness of methotrexate with step-down glucocorticoid remission induction (COBRA Slim) versus other intensive treatment strategies for early rheumatoid arthritis in a treat-to-target approach: 1-year results of CareRA, a randomised pragmatic open-label superiority trial. Ann Rheum Dis 76:511-520

123. Verschueren $P$, De Cock $D$, Corluy $L$ et al (2015) Methotrexate in combination with other DMARDs is not superior to methotrexate alone for remission induction with moderate-to-highdose glucocorticoid bridging in early rheumatoid arthritis after 16 weeks of treatment: the CareRA trial. Ann Rheum Dis 74:27-34

124. Visser K, Van Der Heijde D (2009) Optimal dosage and route of administration of methotrexate in rheumatoid arthritis: a systematic review of the literature. Ann Rheum Dis 68:1094-1099

125. Vital EM, Dass S, Buch MH et al (2015) An extra dose of rituximab improves clinical response in rheumatoid arthritis patients with initial incomplete B cell depletion: a randomised controlled trial. Ann Rheum Dis 74:1195-1201
126. Wailoo A, Hock ES, Stevenson M et al (2017) The clinical effectiveness and cost-effectiveness of treat-to-target strategies in rheumatoid arthritis: a systematic review and cost-effectiveness analysis. Health Technol Assess 21:1-258

127. Weinblatt ME, Fleischmann R, Van Vollenhoven $\mathrm{RF}$ et al (2015) Twenty-eight-week results from the REALISTIC phase IIlb randomized trial: efficacy, safety and predictability of response to certolizumab pegol in a diverse rheumatoid arthritis population. Arthritis Res Ther 17:325

128. Ziegler S, Huscher D, Karberg K et al (2010) Trends in treatment and outcomes of rheumatoid arthritis in Germany 1997-2007: results from the National Database of the German Collaborative Arthritis Centres. Ann Rheum Dis 69:1803-1808

129. Zink A, Manger B, Kaufmann J et al (2014) Evaluation of the RABBIT risk score for serious infections. Ann Rheum Dis 73:1673-1676 